\title{
MORFOANATOMía DEL ÓVULO Y LA SEMILLA EN TURNERA y Piriqueta (Turneraceae)
}

\author{
Ana Maria Gonzalez ${ }^{1}$ y María Mercedes Arbo \\ Instituto de Botánica del Nordeste - CONICET, Corrientes, Argentina \\ ${ }^{1}$ Autor para la correspondencia: anitama39@gmail.com
}

\begin{abstract}
Resumen: Algunos caracteres morfológicos seminales tienen importancia taxonómica y han sido usados para diferenciar géneros y especies en la familia Turneraceae. Sin embargo, su anatomía fue estudiada en pocos taxones. Se analizaron las semillas en los géneros más numerosos de la familia: Piriqueta y Turnera, incluyendo todas las series del último, a fin de completar la información acerca del desarrollo y variantes de los caracteres morfo-anatómicos. Los objetivos fueron caracterizar y constatar su valor taxonómico. Los óvulos presentan anatomía semejante en todas las especies analizadas. Los tegumentos seminales no son multiplicativos, el externo constituye la testa de la semilla, con dos capas de espesor, y el interno forma el tegmen triestratificado. La estructura básica de la cubierta seminal está integrada por aréolas (formadas por células gigantes de la endotesta) limitadas por muros (constituidos por esclereidas del exotegmen), cubiertas por la exotesta, que puede ser lisa o crestada. Algunas especies de Turnera y Piriqueta tienen depresiones punctiformes en las aréolas, solitarias o dobles, determinadas por protrusiones de las células endotestales con paredes laberínticas en la cara interna. La cubierta seminal presenta dos tipos básicos: crestado y reticulado con dos subtipos según el desarrollo de los muros. El subtipo reticulado aparece en Piriqueta y varias series de Turnera; el subtipo estriado-reticulado, con muros transversales poco visibles, en especies de algunas series de Turnera. La cubierta seminal crestada, exclusiva de $T$. sidoides $\mathrm{L}$., se forma cuando las células de la exotesta producen crestas de altura diversa que enmascaran el diseño subyacente. En muchas especies de Piriqueta la exotesta presenta papilas digitifomes, mientras en Turnera aparecen otras papilas. El arilo es hilar en Piriqueta y en la gran mayoría de las especies de Turnera; dos especies de la serie Anomalae y dos de la subserie Umbilicatae presentan arilo ráfico. El género Turnera incluye el 66\% de las especies de la familia, y en concordancia, es el que ofrece mayor diversidad en las semillas.
\end{abstract}

Palabras clave: arilo, cubierta seminal, óvulo, tegmen, testa, Turneraceae.

\begin{abstract}
Some morphological characters of seeds are important in taxonomy of the Turneraceae family, and have been used to distinguish genera and species. However their anatomy has been studied only in a few species. The seeds of Piriqueta and Turnera, the most numerous genera of the family were analyzed -including all the series of the last one- in order to fulfill the information about development and variation of the morph-anatomical characters. The goals were to depict and circumscribe the variants, to check their taxonomic value. The anatomy of ovules is alike in all analyzed species. The integuments are not multiplicative, the outer one makes up the seed testa, with two layers, and the inner integument develops the tegmen with three layers. The seed coat basic structure is composed of areoles (made up by the giant cells of endotesta) delimited by muri (set out by exotegmen sclereids). Some species of Turnera and Piriqueta have one or two dot-like hollows in each areole, set up by protrusions of the endotesta cells with labyrinth walls on the inner face. The seed coat shows two basic types: reticulate and crested. According to the degree of development of transverse muri, the reticulate type shows two subtypes. The most common is the reticulate subtype, found in Piriqueta and in several series of Turnera. The striate-reticulate subtype, with transverse muri hardly visible, is found in species of some series of Turnera. The crested seed coat, found exclusively in T. sidoides, comes out when groups of exotesta cells grow developing crests of different height, which cover and hide the basic design. In many species of Piriqueta, the exotesta shows finger-like papillae while in Turnera it displays other types of papillae. The aril is inserted around the hilum in Piriqueta and in most species of Turnera, but in two species of series Anomalae and two of subseries Umbilicatae it is inserted also along the basal segment of the raphe. Turnera includes $66 \%$ of the species of the family, and in agreement it shows the towering diversity in seed morphology.

Key words: aril, ovule, seed coat, tegmen, testa, Turneraceae.
\end{abstract}

L a familia Turneraceae cuenta con 227 especies asignadas a 12 géneros, ocho distribuidos en África, Madagascar e islas Mascareñas (Thulin et al., 2012), tres en América (Piriqueta (44 spp.), Adenoa (1 spp.) y Erblichia (1 spp.), y Turnera con 141 especies en América y dos en África, repartidas en 11 series: Annulares (4), Anomalae (14), Capitatae (10), Conciliatae (1), Leiocarpae (56), Microphyllae (5), Papilliferae (2), Salicifoliae (12), Sessilifoliae (2), Ste- 
nodictyae (10), y Turnera, con dos subseries: Turnera (19) y Umbilicatae (8). Turnera y Piriqueta abarcan $80 \%$ de las especies de la familia (Arbo 1995, 1997, 2000, 2005, 2008; Grande et al., 2012).

La morfología de las semillas ha sido tratada en las revisiones taxonómicas (Urban, 1883; Arbo, 1995, 1997, 2000, 2005, 2008). Los estudios anatómicos se refieren a unas pocas especies: Berger (1919) describe e ilustra la cubierta seminal de Piriqueta racemosa (Jacq.) Sw. y varias especies de Turnera. Corner (1976), basándose en los trabajos de Raju (1956) y Vijayaraghavan y Kaur (1967) acerca de T. ulmifolia L., generalizó que la familia tiene semillas reticuladas, con arilo funicular, procedentes de óvulos anátropos, bitégmicos con tegumento externo biestratificado y tegumento interno de tres capas, no multiplicativos, testa no especializada y tegmen con un estrato de células esclerosadas. Los estudios ontogenéticos de las semillas se limitan a T. ulmifolia (Raju, 1956; Vijayaraghavan y Kaur, 1967). Bahadur et al. (1981) compararon la cubierta seminal y el arilo en T. ulmifolia y T. subulata Sm. Kloos y Bouman (1980) estudiaron la ontogenia del arilo en Passiflora suberosa L. y T. ulmifolia, y lo definieron como ráfico. Gonzalez (1993) estudió los óvulos de Piriqueta racemosa y dos especies de Turnera.

Teniendo en cuenta la escasez de datos en relación al número de especies, los objetivos de este trabajo fueron: caracterizar la variabilidad morfoanatómica de los caracteres seminales en Piriqueta y Turnera, cotejar su valor taxonómico y describir los cambios acontecidos durante la ontogenia de la cubierta seminal y del arilo.

\section{Material y métodos}

El estudio incluyó 11 especies de Piriqueta y 55 especies de Turnera abarcando todas sus series y subseries. La lista de ejemplares que indica coleccionista, número, procedencia y herbarios consultados figura en el Apéndice.

Las semillas, flores y frutos en distintos estadíos de desarrollo se fijaron en FAA (alcohol etílico 70\%, formol 5\% y ácido acético 5\%, 90:5:5); se deshidrataron e incluyeron en parafina (Johansen, 1940; Gonzalez y Cristóbal, 1997). Se realizaron cortes seriados transversales (CT) y longitudinales (CL), de 10-12 $\mu \mathrm{m}$ y se tiñeron con safranina y Astra blue (Luque et al., 1996). Se utilizó IKI-ácido sulfúrico (Jensen, 1962) y sulfato ferroso (Ruzin, 1999) para la identificación de taninos; floroglucinol para celulosa/lignina (D’Ambrogio de Argüeso, 1986); el almidón se identificó con luz polarizada y tinción con lugol (Johansen, 1940).

Para el estudio con Microscopio Electrónico de Barrido (MEB), los óvulos y semillas jóvenes fueron deshidratados en serie acetónica creciente, secados a punto crítico y cubiertos con oro-paladio. Se utilizó el equipo Jeol 5800 LV a $20 \mathrm{kV}$ del Servicio de Microscopía Electrónica de Barrido de la Universidad Nacional del Nordeste, Corrientes. Algunas fotografías fueron tomadas en Washington University,
St. Louis, EE.UU. y York University, Toronto, Canadá.

Para las descripciones anatómicas se empleó la terminología de Barthlott $(1981,1984)$, con modificaciones cuando fue necesario. Se utilizó la Tabla de Munsell (1977) para describir los colores de las semillas maduras.

\section{Resultados}

Ontogenia de los óvulos. Las flores de ambos géneros presentan ovario súpero, sincárpico, tricarpelar, unilocular con placentación parietal; los óvulos son anátropos y se disponen en abanico en cada placenta linear (Figura 1) situada en una sutura carpelar. Los primordios seminales se inician como protuberancias hemisféricas originadas por divisiones periclinales de los estratos dérmico y subepidérmico de las placentas (Figuras 1A-B, 2A-B). Cada primordio se alarga por crecimiento del funículo y el ápice se curva (Figura 1C, E). Los tegumentos se originan por divisiones anticlinales y oblicuas del estrato dérmico en zonas anulares alrededor de la nucela. El tegumento interno se forma primero (Figuras 1D-F, 2C-D); el tegumento externo aparece como un anillo interrumpido sobre la rafe (Figura 2C-E). Los óvulos son crasinucelados, la célula madre de las megásporas se diferencia 3-4 capas por debajo de la epidermis nucelar unistrata (Figura 2F).

Cuando el óvulo completa el giro de $180^{\circ}$ se diferencia, por actividad del estrato dérmico, la porción de tegumento externo faltante entre tegumento interno y rafe, (Figura $2 \mathrm{~F}$ ); este crece rápidamente y forma el exóstoma antes que el tegumento interno alcance el ápice nucelar. Así queda el exóstoma orientado hacia el funículo y el micrópilo en zigzag (Figura 2G, I). La capa interna del tegumento interno sufre divisiones, quedando éste constituido por tres estratos (Figura $2 \mathrm{H}, \mathrm{J}$ ). La rafe posee un haz vascular que llega hasta la cálaza, y presenta células taníferas en las especies de $P i$ riqueta (Figura 2F-G). La epidermis de funículo y placentas se diferencia en tejido de transmisión (Figura 2G, I).

En el óvulo maduro, la epidermis del tegumento externo tiene células con abundantes compuestos tánicos y estomas anomocíticos simples, dobles y triples. El tegumento interno tiene tres estratos. En algunas especies el exóstoma y el endóstoma son muy prominentes, formando un "pico" curvado hacia el funículo (Figura 1H, I).

El saco embrionario sólo se observó claramente en Turnera joelii, siendo de tipo Polygonum. Las células de la cálaza forman una prominencia más o menos globosa (Figuras $1 \mathrm{G}, \mathrm{H}, 2 \mathrm{G}, \mathrm{I})$.

Semilla. Las semillas maduras son negras a castaño-oscuras $(10$ YR $2 / 1$ a 10 YR 3/2) y pequeñas, miden 1.5-4.90 × 0.7$2.7 \mathrm{~mm}$. En vista rafeal son obovoides o elipsoides, en vista lateral (con la rafe a un lado) son curvadas o rectas (Figura 3C, M); en contorno pueden ser virguliformes (Figura 3G), obovoides (Figura $3 \mathrm{H}$ ), subglobosas (Figura $3 \mathrm{C}$ ) o raramen- 

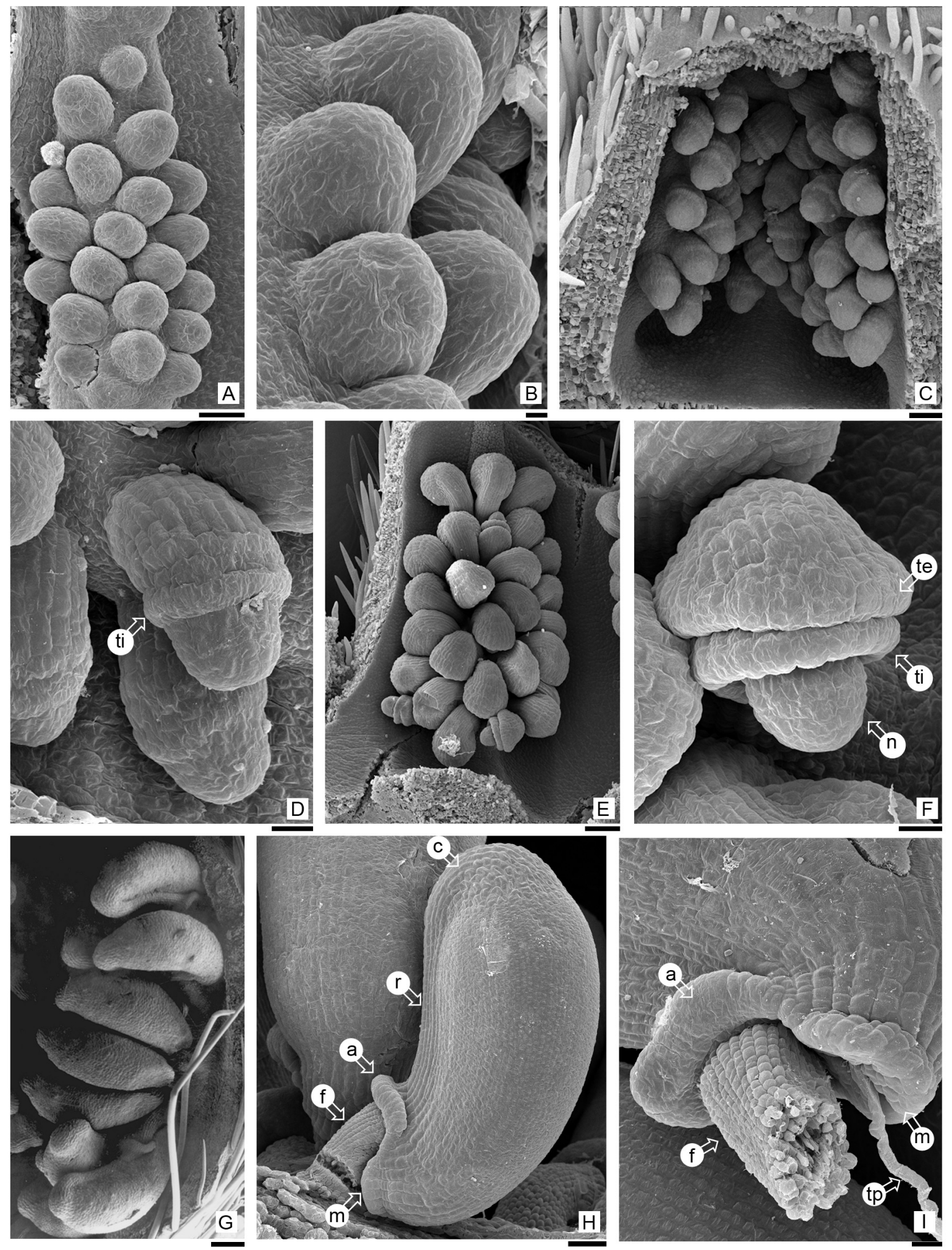

Figura 1. Ontogenia de óvulos (MEB). A-F. Piriqueta suborbicularis. G-I. Turnera weddelliana. A-B. Placenta y detalle de óvulos en desarrollo, estadío sin tegumentos. C-D. CL de ovario con placenta y óvulos al inicio de tegumento interno. E-F. Placenta y óvulos con ambos tegumentos. G. Placenta con óvulos anátropos. H. Óvulo anátropo con arilo incipiente. I. Detalle de la zona micropilar mostrando la penetración de un tubo polínico y la inserción del arilo. a: arilo; c: cálaza; f: funículo; m: micrópilo; n: nucela; r: rafe; te: tegumento externo; ti: tegumento interno; tp: tubo polínico. Barras A, C, F, H=50 $\mu \mathrm{m} ; \mathrm{B}=10 \mu \mathrm{m} ; \mathrm{D}, \mathrm{I}=20 \mu \mathrm{m} ; \mathrm{E}, \mathrm{G}=100 \mu \mathrm{m}$. 

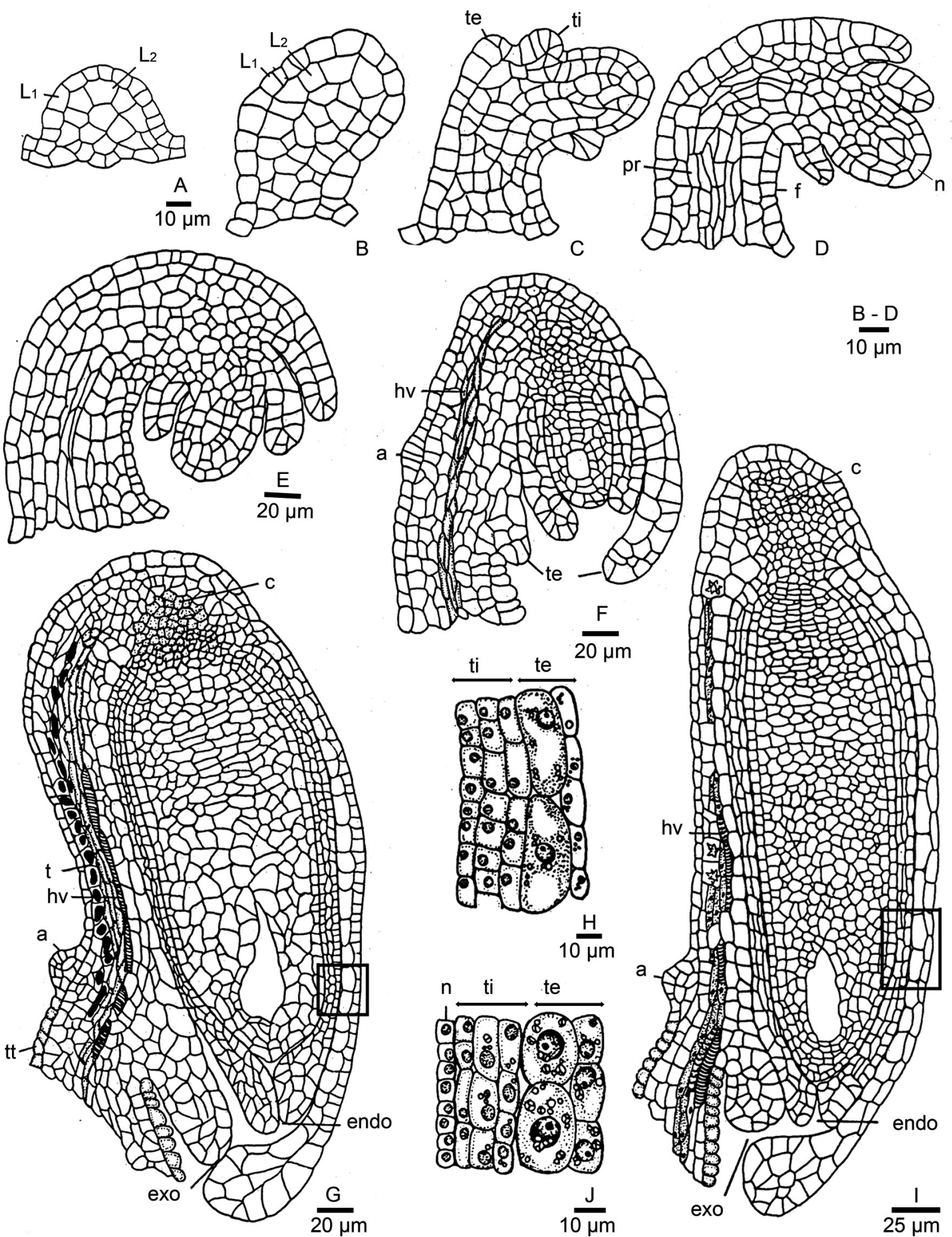

$10 \mu \mathrm{m}$ 
te hemisféricas (Turnera lineata). En vista lateral la rafe puede ser cóncava (Figura 3D), recta, convexa o sigmoide (Figura 3K), pero el lado opuesto siempre es convexo; la rafe, usualmente recta, a veces es sigmoide (T. luetzelburgii); en el extremo apical, llegando a la cálaza, puede presentar una proliferación de células epidérmicas formando un engrosamiento elíptico o circular (Figura 3E-F).

Las semillas presentan exóstoma notable, con la base marcada por un resalto (Figura $3 \mathrm{~K}$ ) o una cresta exostomal (Figuras 3N-O, 7C). La cálaza puede ser obtusa (Figura 3A, B, G), prominente (Figura 3K-L) o cónica (Figura 3D), y su superficie varía de plana a cóncava; cuando es prominente y cóncava se forma un reborde que la vuelve umbilicada (Figura 3K, 9O); a veces presenta un ligero estrechamiento en la base (Figura 3K-L).

Los caracteres seminales de las especies estudiadas de Piriqueta y Turnera se encuentran resumidos en el cuadro 1.

La cubierta seminal de Turnera y Piriqueta está constituida por diferenciación de ambos tegumentos (Figuras 4AC, 5A-C). El tegumento externo del óvulo origina la testa: el estrato dérmico forma la exotesta y el subepidérmico la endotesta. El tegmen se forma a partir de los tres estratos del tegumento interno del óvulo, pero sólo el exotegmen presenta diferenciación en la semilla madura, transformándose en su estrato mecánico.Se presentan en dos tipos básicos: reticulado (Figura 3A-M) y crestado (Figura 3N-P), que resultan de la combinación de las características anatómicas y el grado de desarrollo de exotesta, endotesta y exotegmen.

(1) Cubierta seminal reticulada. Presenta muros longitudinales y transversales formados por el exotegmen y aréolas demarcadas por células de la endotesta. La exotesta acompaña el diseño de las capas subyacentes. De acuerdo al predominio de los muros se subdivide en: (1.1) reticulado, todos los muros que rodean las aréolas tienen igual desarrollo (Figura 3A-G, I-J); y (1.2) estriado/reticulado, predominan los muros longitudinales, los transversales son poco visibles (Figura 3H, K-M).

(2) Cubierta seminal crestada. La escultura es determinada principalmente por la exotesta o epidermis, cuyas células forman crestas de diversa altura. La endotesta y el exotegman forman muros y aréolas como en el diseño reticulado, pero quedan ocultos bajo las crestas de la exotesta. Es exclusiva de Turnera sidoides (Figura $3 \mathrm{~N}-\mathrm{Q}$ ), con cinco subespecies.

Anatomía de la cubierta seminal. Semillas reticuladas. Exotesta: constituida por un estrato de células alargadas y tabulares en CT (Figura 4F). La pared tangencial externa puede ser (1) recta, plana (Figura 6A); (2) convexa o con domos (Figura 6B, J) y (3) con papilas diversas. (3.1) Filiformes: relación largo/ancho $>8: 1$; la base de la papila es menor que el ancho de la célula, son cortas de hasta $0.09 \mathrm{~mm}$ (Figura 6D) o largas de 0.1 a $0.28 \mathrm{~mm}$ (Figuras 4C, 6E); pueden ser tan abundantes que la semilla parece pilosa, aún a ojo desnudo (Figura 3H). (3.2) Digitiformes: la base de la papila es menor que la pared externa de la célula, relación largo/ ancho < 5:1 (Figuras 6F-G, L, 7F); se ven con el máximo aumento del microscopio estereoscópico, pero sólo se pueden medir con MEB. (3.3) Mamiliformes: cada célula posee una papila conoidal cuya base es toda la pared externa de la célula y no supera las 15-20 $\mu \mathrm{m}$ de altura (Figura 6H-I). (3.4) Hemisféricas: la base de la papila puede ocupar todo el ancho de la célula o ser menor (Figura $6 \mathrm{C}, \mathrm{K}$ ) y su altura es igual que en las anteriores.

En Turnera weddelliana algunas células presentan paredes convexas y otras tienen papilas filiformes largas (Figura 6E). En ciertas especies pueden presentarse adcrustaciones de ceras en forma de delgados filamentos (Figuras 5M, 6K).

Endotesta: es uno de los estratos que más cambio sufre durante la diferenciación de la semilla, forma células gigantes que determinan las aréolas del retículo; poseen núcleo muy grande, con varios nucléolos, granos de almidón simples y compuestos (Figuras 3, 4, 5). En transcorte, estas células varían, determinando diseños acordes en la superficie de la semilla: (1) células elípticas, lenticulares, hemisféricas, conoidales o con forma de escudo, que al desecarse producen aréolas con fondo cóncavo (Figuras 4G-H, 5A, F); (2) células mamiliformes, la pared tangencial interna presenta una porción hemisférica que se incrusta entre las células del exotegmen (Figuras 4C-D, 5B-D), que forma una depresión punctiforme en el fondo de la aréola (Figura 5I-J). (3) En algunas especies la pared interna posee dos porciones hemisféricas, sólo observables en CT de semilla (Figura 5E), que producen dos depresiones punctiformes en cada aréola.

En Piriqueta, la pared interna presenta engrosamientos laberínticos que se proyectan hacia el lumen celular y se impregnan de sustancias tánicas (Figura 5C-D). Las células de la endotesta están dispuestas en hileras longitudinales, ocasionalmente se encuentran desordenadas, como en $\mathrm{P}$. $\mathrm{ra}$ cemosa (Figura 3A) o Turnera trigona (Figura 3F).

Exotegmen: las células crecen radialmente y se orientan de forma oblicua, curvándose hacia afuera y en dirección al micrópilo (Figura 4). Desarrollan esclereidas de pared muy gruesa y lignificada, con puntuaciones simples y ramificadas; el lumen se reduce y puede llegar a obliterarse, a la madurez presenta sustancias tánicas (Figura 4E). Su forma final puede ser alargada, deltoide, triangular o romboidal, ya que se amoldan a la forma de las células de la endotesta (Figuras 4D, F, H, 5A-C). Varían también en altura, algunas se encajan entre las células de la endotesta y pueden alcanzar la exotesta, forman los muros y nudos del retículo en la cubierta seminal; las más bajas componen la base de las aréolas. En Turnera guianensis, T. nervosa, T. pumilea y $T$. trigona los nudos son salientes, ya que las esclereidas son más altas en las zonas de unión de tres o cuatro aréolas (Figura 3D). En Piriqueta racemosa los nudos son tan prominentes que los muros resultan poco visibles (Figura 3A).

En el endóstoma las esclereidas son rectas y paralelas, pueden tener la misma altura que en los lados de la semilla o 
Cuadro 1: Caracteres seminales en las especies de Piriqueta y Turnera estudiadas.

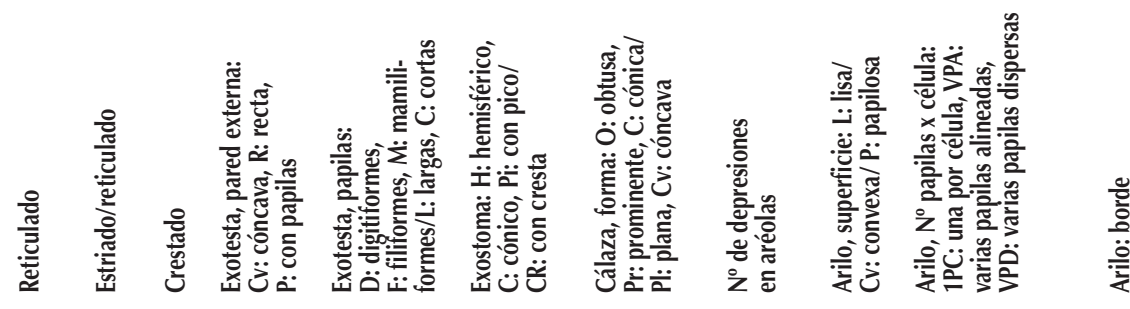

\section{Piriqueta}

Piriqueta carnea

Piriqueta cistoides

Piriqueta densiflora var. densiflora

Piriqueta caiapoensis

Piriqueta duarteana var. duarteana

Piriqueta morongii

Piriqueta nanuzae

Piriqueta ochroleuca

Piriqueta racemosa

Piriqueta sidifolia var. multiflora

Piriqueta suborbicularis

Piriqueta taubatensis

\section{Serie ANOMALAE}

Turnera blanchetiana

Turnera cearensis

Turnera laciniata

\section{Serie ANNULARES}

Turnera annularis

Turnera aromatica

Turnera breviflora

Turnera odorata

\section{Serie CAPITATAE}

Turnera albicans

Turnera capitata

\section{Serie CONCILIATAE}

Turnera rubrobracteata

\section{Serie LEIOCARPAE}

Turnera argentea

Turnera cipoensis

Turnera coccinea

Turnera dasytricha

Turnera discolor

Turnera genistoides

Turnera guianensis

Turnera hassleriana

Turnera lamiifolia

Turnera longiflora

Turnera melochioides

Turnera nervosa

Turnera opifera

M-H
$M$
$H$

$\mathrm{H}$

M

$x$

$x$

$x$

$x$

X

$x$

$\mathrm{X}$

R

\begin{tabular}{|c|c|}
\hline Cv-P & $\mathrm{H}$ \\
\hline R & \\
\hline$R$ & \\
\hline $\mathrm{R} / \mathrm{P}$ & $\mathrm{H}$ \\
\hline$R$ & \\
\hline $\mathrm{Cv}$ & \\
\hline$R$ & \\
\hline Cv-P & $\mathrm{H}$ \\
\hline $\mathrm{R}-\mathrm{CV}$ & \\
\hline $\mathrm{Cv}$ & \\
\hline $\mathrm{Cv}$ & \\
\hline$P$ & $\mathrm{H}$ \\
\hline$R$ & \\
\hline
\end{tabular}

$\begin{array}{cccccc}\mathrm{H} & \mathrm{O}-\mathrm{Pr} / \mathrm{Pl}-\mathrm{CV} & 0 & \mathrm{P} & \text { VPD } & \text { Lobulado } \\ \mathrm{C} & \mathrm{Pr} / \mathrm{Pl}-\mathrm{CV} & 0 & \mathrm{~L} & - & \text { Ent./ Lob. } \\ \mathrm{H} & \mathrm{Pr} / \mathrm{Pl}-\mathrm{CV} & 0 & \mathrm{~L} & - & \text { Lob./ Lacin. } \\ \mathrm{C} & \mathrm{Pr} / \mathrm{Pl}-\mathrm{CV} & 0 & \mathrm{~L} & - & \text { Ent./ Lob. }\end{array}$

$\mathrm{HC}$

$\mathrm{Pr} / \mathrm{CV}$

$\mathrm{Pr} / \mathrm{Pl}$

$\mathrm{Pr} / \mathrm{CV}$

$\mathrm{Pr} / \mathrm{Cv}$

$\begin{array}{ll}0 & \mathrm{~L} \\ 0 & \mathrm{CV}\end{array}$

Lobulado

Lobulado

Laciniado

Lobulado

VPA Laciniado

VPA Laciniado 


\begin{tabular}{|c|c|c|c|c|c|c|c|c|c|c|c|}
\hline & $\frac{\stackrel{ }{\frac{E}{ \pm}}}{\bar{E}}$ & 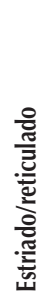 & 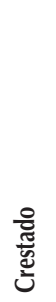 & 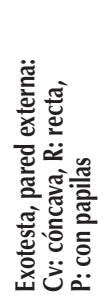 & 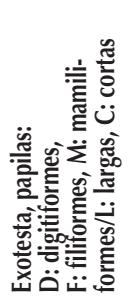 & 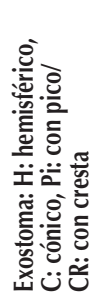 & 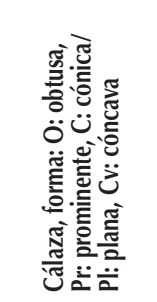 & 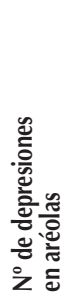 & 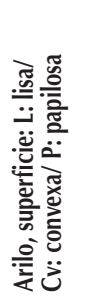 & 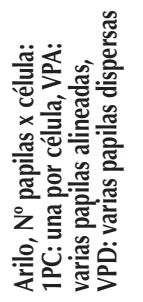 & $\frac{\text { 苛 }}{\frac{0}{\ddot{0}}}$ \\
\hline Turnera pumilea & $\mathrm{X}$ & & & $\mathrm{R}$ & & $\mathrm{H}$ & $\mathrm{Pr} / \mathrm{Pl}$ & 0 & $\mathrm{~L}$ & & Entero \\
\hline Turnera sidoides subsp. carnea & & & $x$ & $\mathrm{R} / \mathrm{P}$ & $\mathrm{D}$ & $\mathrm{C} / \mathrm{CR}$ & $\mathrm{O}-\mathrm{Pr} / \mathrm{PI}-\mathrm{Cv}$ & 0 & $P$ & VPA & Lobulado \\
\hline Turnera sidoides subsp. integrifolia & & & $\mathrm{X}$ & $\mathrm{R} / \mathrm{P}$ & $\mathrm{D}$ & $\mathrm{C} / \mathrm{CR}$ & $\mathrm{O} / \mathrm{Pl}$ & 0 & $P$ & - & Lobulado \\
\hline Turnera sidoides subsp. pinnatifida & & & $x$ & $\mathrm{R} / \mathrm{P}$ & $\mathrm{D}$ & $\mathrm{C} / \mathrm{CR}$ & $\mathrm{O} / \mathrm{PI}$ & 0 & $P$ & - & Entero \\
\hline Turnera sidoides subsp. sidoides & & & $\mathrm{X}$ & $\mathrm{R} / \mathrm{P}$ & $\mathrm{D}$ & $\mathrm{C} / \mathrm{CR}$ & $\mathrm{O} / \mathrm{Pl}$ & 0 & $P$ & VPA & Entero \\
\hline Turnera subnuda & $\mathrm{X}$ & & & $\mathrm{R} / \mathrm{P}$ & $\mathrm{H}$ & $\mathrm{C}$ & $\mathrm{Pr} / \mathrm{PI}$ & $0-1$ & L & & Lobulado \\
\hline Turnera trigona & $X$ & & & $\mathrm{R} / \mathrm{P}$ & $\mathrm{H}$ & $\mathrm{H}$ & $\mathrm{Pr} / \mathrm{Pl}-\mathrm{Cv}$ & 0 & $\mathrm{Cv}$ & & Lobulado \\
\hline \multicolumn{12}{|l|}{ Serie MICROPHYLLAE } \\
\hline Turnera collotricha & $X$ & & & $\mathrm{R}$ & & $\mathrm{H}$ & $\mathrm{O} / \mathrm{PI}$ & 0 & $P$ & VPA & Entero \\
\hline Turnera diffusa & $X$ & & & $\mathrm{R}$ & & $\mathrm{H}$ & $\mathrm{O} / \mathrm{Pl}$ & 1 & $P$ & VPA & Entero \\
\hline Turnera hebepetala & $X$ & & & $\mathrm{R}$ & & $\mathrm{C}$ & $\mathrm{O} / \mathrm{PI}$ & 1 & $\mathrm{Cv}$ & & Entero \\
\hline \multicolumn{12}{|l|}{ Serie PAPILLIFERAE } \\
\hline Turnera caatingana & $X$ & & & $\mathrm{R}$ & & $\mathrm{C}$ & $\mathrm{Pr} / \mathrm{Pl}$ & 0 & L & & Lobulado \\
\hline Turnera chamaedrifolia & $x$ & & & $\mathrm{R}$ & & $\mathrm{H}$ & $\mathrm{O} / \mathrm{Pl}$ & 0 & L & & Lobulado \\
\hline \multicolumn{12}{|l|}{ Serie SALICIFOLIAE } \\
\hline Turnera brasiliensis & & $X$ & & $P$ & $\mathrm{~F} / \mathrm{L}$ & $\mathrm{Pi}$ & $\mathrm{Pr} / \mathrm{Cv}$ & 0 & L & & Lobulado \\
\hline Turnera glaziovii & & $\mathrm{X}$ & & $\mathrm{CV}_{-} \mathrm{P}$ & $\mathrm{F} / \mathrm{L}$ & $\mathrm{Pi}$ & $\mathrm{Pr} / \mathrm{Cv}$ & 0 & $\mathrm{Cv}$ & & Lobulado \\
\hline Turnera hindsiana & $x$ & & & $P$ & $\mathrm{H}-\mathrm{F} / \mathrm{L}$ & $\mathrm{Pi}$ & $\mathrm{Pr} / \mathrm{Cv}$ & 0 & L & & Lobulado \\
\hline Turnera panamensis & & $\mathrm{X}$ & & $\mathrm{CV}_{\mathrm{V}} \mathrm{P}$ & $\mathrm{F} / \mathrm{L}$ & $\mathrm{Pi}$ & $\mathrm{Pr} / \mathrm{Cv}$ & 0 & L & & Lobulado \\
\hline Turnera rupestris & & $X$ & & Cv-P & $\mathrm{F} / \mathrm{L}$ & $\mathrm{Pi}$ & $\mathrm{Pr} / \mathrm{Cv}$ & 0 & L & & Lobulado \\
\hline Turnera serrata & & $\mathrm{X}$ & & $\mathrm{P}$ & $\mathrm{H}-\mathrm{F} / \mathrm{L}$ & $\mathrm{Pi}$ & $\mathrm{Pr} / \mathrm{Cv}$ & 1 & $P$ & VPA & Entero \\
\hline Turnera venosa & & $\mathrm{X}$ & & $\mathrm{CV}_{\mathrm{V}} \mathrm{P}$ & $\mathrm{F} / \mathrm{L}$ & $\mathrm{Pi}$ & $\mathrm{Pr} / \mathrm{Cv}$ & 0 & L & & Lobulado \\
\hline Turnera weddelliana & & $X$ & & $P$ & $\mathrm{H}-\mathrm{F} / \mathrm{L}$ & $\mathrm{Pi}$ & $\mathrm{Pr} / \mathrm{Cv}$ & 0 & $\mathrm{Cv}$ & & Lobulado \\
\hline \multicolumn{12}{|l|}{ Serie SESSILIFOLIAE } \\
\hline Turnera dichotoma & $x$ & & & $\mathrm{R}$ & & $\mathrm{H}$ & $\mathrm{O} / \mathrm{PI}$ & 1 & $P$ & VPA & Lobulado \\
\hline Turnera revoluta & & $\mathrm{X}$ & & $\mathrm{R}$ & & $\mathrm{H}$ & $\mathrm{O} / \mathrm{Pl}-\mathrm{Cv}$ & 0 & L & & Entero \\
\hline \multicolumn{12}{|l|}{ Serie STENODICTYAE } \\
\hline Turnera acuta & & $\mathrm{X}$ & & $\mathrm{P}$ & $\mathrm{D}$ & $\mathrm{C}$ & $\mathrm{Pr} / \mathrm{Cv}$ & 0 & $\mathrm{Cv}$ & & Laciniado \\
\hline Turnera aurantiaca & $x$ & & & $\mathrm{P}$ & $\mathrm{F} / \mathrm{C}$ & $\mathrm{C}$ & $\mathrm{Pr} / \mathrm{Cv}$ & 0 & L & & Lobulado \\
\hline \multicolumn{12}{|l|}{ Serie TURNERA, subserie Turnera } \\
\hline Turnera aurelii & $x$ & & & $\mathrm{R}$ & & $\mathrm{H}$ & $\mathrm{Pr} / \mathrm{Cv}$ & 0 & $\mathrm{Cv}$ & & Lobulado \\
\hline Turnera grandiflora & $x$ & & & $\mathrm{R}$ & & $\mathrm{H}$ & $\mathrm{O} / \mathrm{Pl}$ & 0 & $\mathrm{Cv}$ & & Lobulado \\
\hline Turnera scabra & $x$ & & & $\mathrm{R}$ & & $\mathrm{C}$ & $\mathrm{O} / \mathrm{PI}$ & 0 & $\mathrm{Cv}$ & & Lobulado \\
\hline \multicolumn{12}{|l|}{ Serie TURNERA, subserie Umbilicatae } \\
\hline Turnera coriacea & $x$ & & & $\mathrm{R}$ & & $\mathrm{C}$ & $\mathrm{Pr} / \mathrm{Cv}$ & 0 & $P$ & VPA & Lobulado \\
\hline Turnera hermannioides & $x$ & & & $\mathrm{R}$ & & $\mathrm{H}$ & $\mathrm{Pr} / \mathrm{Cv}$ & 0 & $P$ & VPA & Lobulado \\
\hline Turnera joelii & $x$ & & & $\mathrm{R}$ & & $\mathrm{H}$ & $\mathrm{Pr} / \mathrm{Cv}$ & $0-1$ & $\mathrm{Cv}$ & & Lobulado \\
\hline Turnera leptosperma & $x$ & & & $\mathrm{R}$ & & $\mathrm{H}$ & $\mathrm{Pr} / \mathrm{Cv}$ & 0 & $\mathrm{Cv}$ & & Lobulado \\
\hline Turnera stenophylla & $x$ & & & $\mathrm{R}$ & & $\mathrm{C}$ & $\mathrm{Pr} / \mathrm{Cv}$ & 0 & $P$ & 1PC-VPA & Laciniado \\
\hline
\end{tabular}



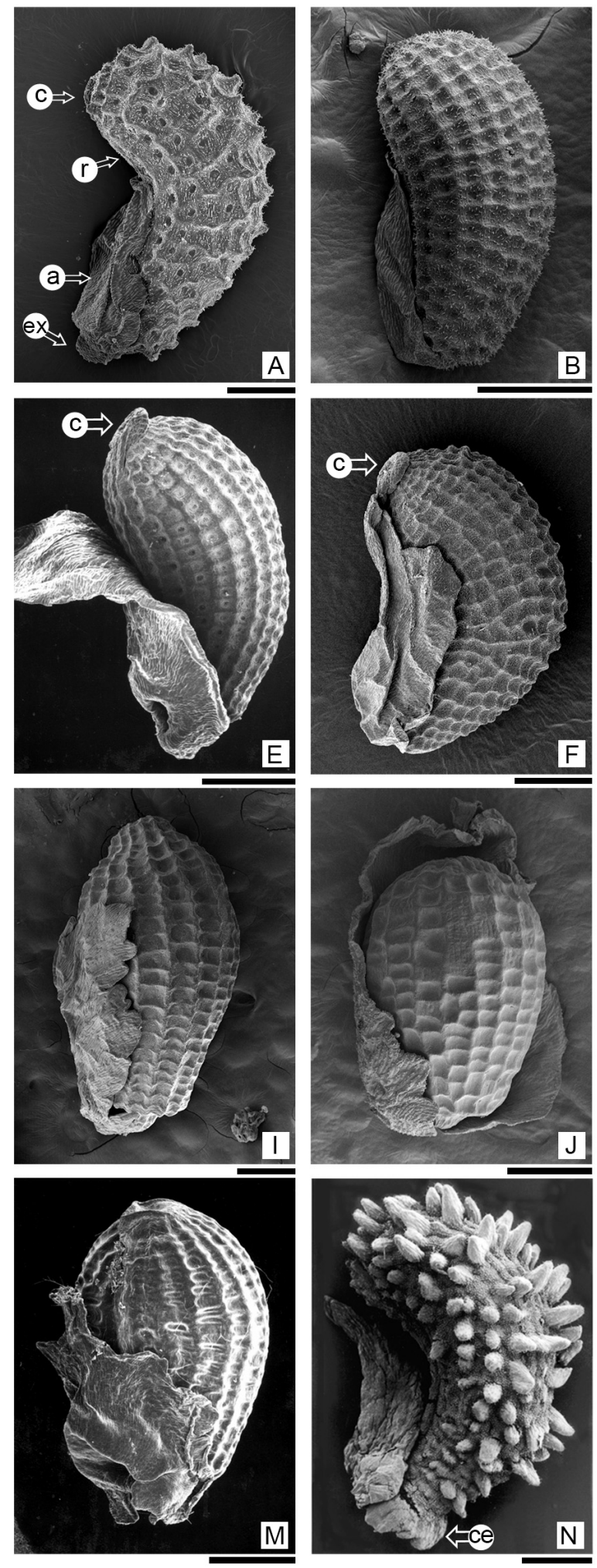
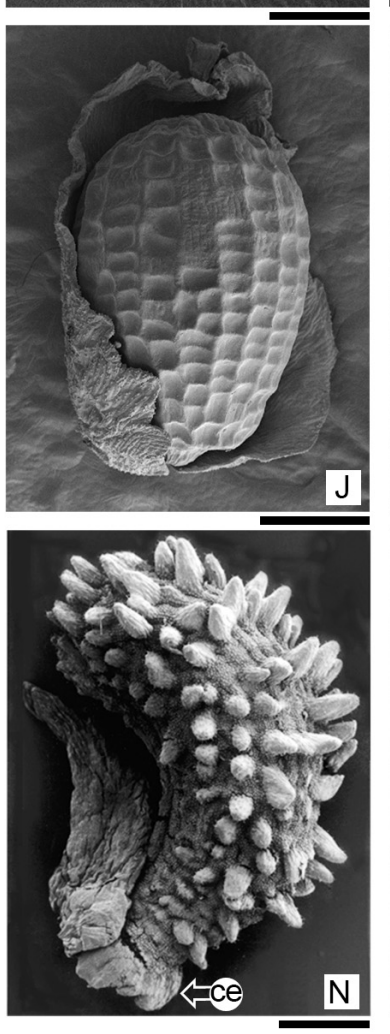
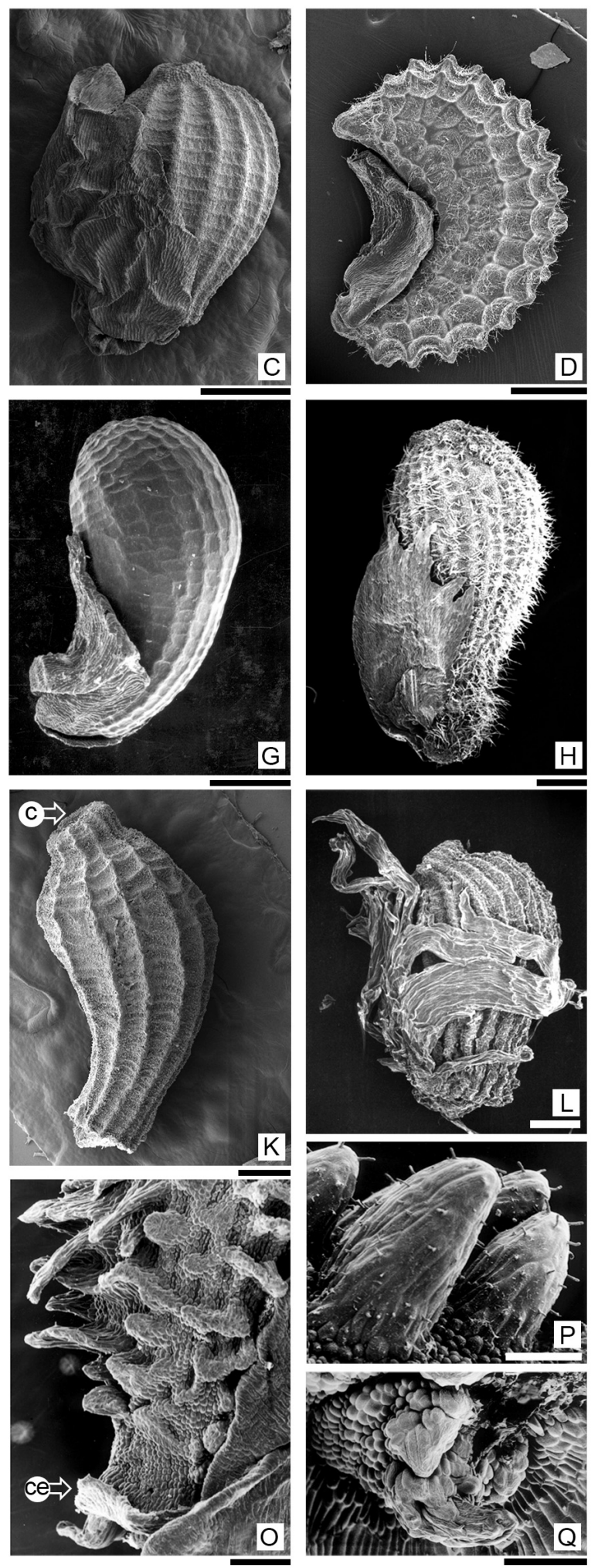

Figura 3. Semillas maduras con MEB. A. Piriqueta racemosa. B. P. cistoides. C. Turnera aromatica. D. T. pumilea. E. T. dasytricha. . T. trigona. G. T. collotricha. H. T. hindsiana. I. T. capitata. J. T. chamaedryfolia. K. T. acuta (sin arilo). L. T. laciniata. M. T. collotricha. N-Q. T. sidoides subsp. pinnatifida. O. Detalle de la región basal con cresta exostomal anular. P. Detalle de la cubierta seminal crestada. Q. Detalle del hilo. a: arilo; c: cálaza; ce: cresta exostomal anular; ex: exóstoma; r: rafe.Barras: A-N =0.5 mm; O = $200 \mu \mathrm{m} ; \mathrm{P}-\mathrm{Q}=$ $20 \mu \mathrm{m}$. 

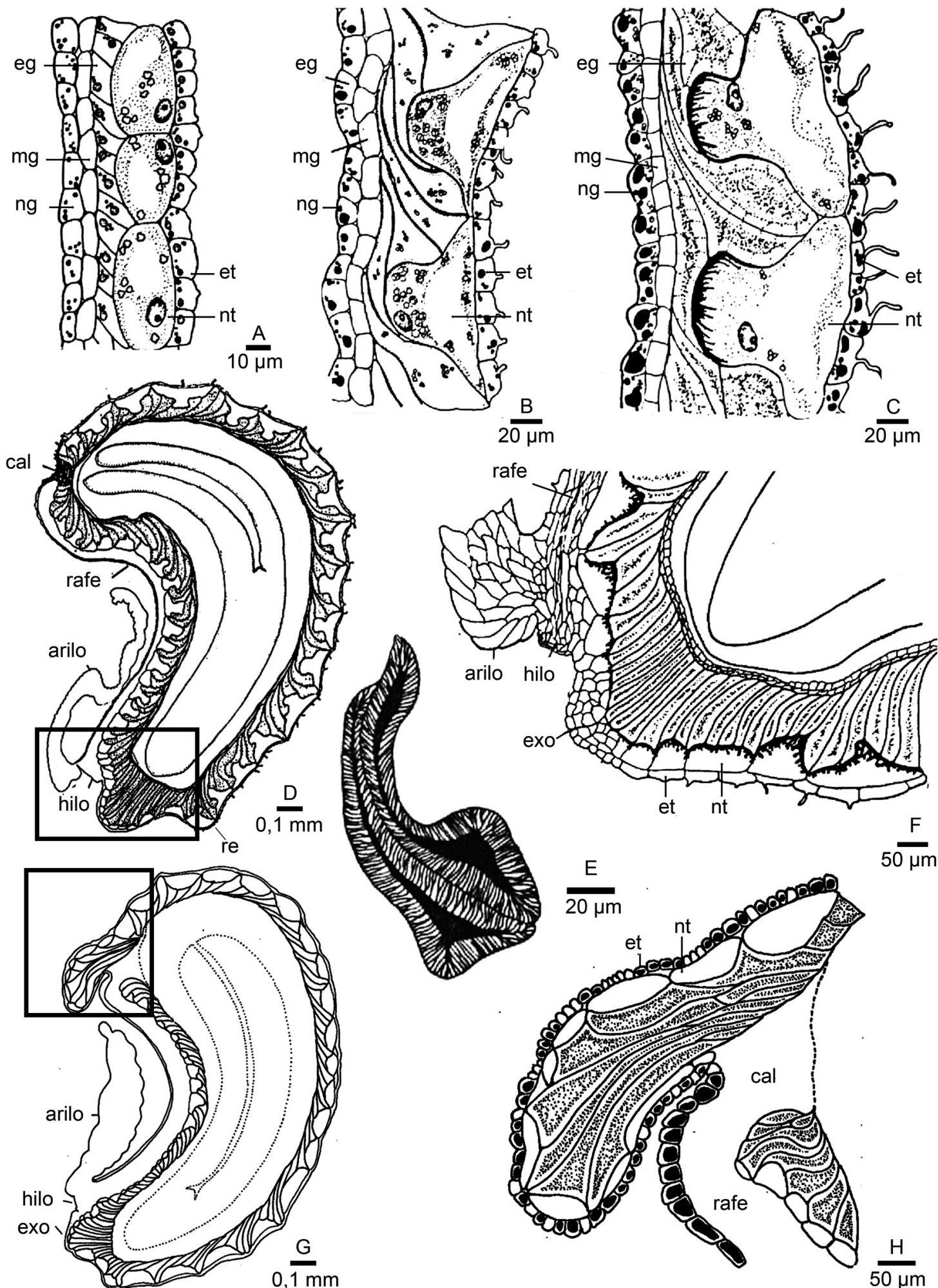

Figura 4. Esquemas de cubierta seminal en secciones longitudinales de semilla. A-E. Piriqueta racemosa. A-C. Sucesivos estados de desarrollo de la cubierta seminal. D. CL de semilla madura. E. Detalle de una esclereida del exotegmen. F. P. morongii, detalle de la región de la micrópila de la semilla madura mostrando organización de exóstoma, hilo e inserción del arilo. G-H. T. pumilea. G. Semilla madura. H. Detalle de la región de la cálaza. cal: cálaza; eg: exotegmen; endo: endóstoma; et: exotesta; exo: exóstoma; mg: mesotegmen; ng: endotegmen; 
ser más altas formando un prominente pico, orientado hacia el funículo (Figuras 4F, 5M). En la base del endóstoma, las primeras esclereidas que se curvan hacia afuera forman un resalto que puede ser más o menos conspicuo (Figuras 3I-K, 4D, G, 5L-M). Las esclereidas se interrumpen en la cálaza, en algunas especies las últimas esclereidas pueden alargarse formando un reborde (Figura 5J); en Turnera acuta el tejido ubicado entre esas esclereidas es parenquimático, y cuando se deseca la cálaza toma forma umbilicada (Figura 3K); en T. pumilea las esclereidas forman una conspicua prominencia cónica (Figuras 3D; 4G-H).

Mesotegmen y endotegmen: células pequeñas, cuadrangulares a rectangulares en $\mathrm{CT}$, a veces con una gran vacuola tánica (Figuras 4A-C, 5B, 7D-F). En la semilla madura estos estratos prácticamente no sufren modificaciones y son comprimidos por los externos (Figura 5A-B)

Semillas crestadas. Exotesta: durante la ontogenia ciertas células de la exotesta se alargan radialmente (Figura 7), diferenciándose grupos de células altas que forman crestas horizontales o verticales, de hasta $250 \mu \mathrm{m}$ de altura. Algunas células presentan una papila digitiforme, de 10-12 $\mu \mathrm{m}$, sin lumen, formada sólo por pared y cutícula (Figura 6L). En el citoplasma se depositan taninos. Los estomas frecuentemente quedan intercalados entre las células que no se alargan (Figura 6K). Endotesta: las células adquieren forma elipsoidal en transcorte; en corte paradermal son cuadrangulares; tienen paredes celulósicas, delgadas, granos de almidón y núcleo grande (Figura 5F). Exotegmen: en la base del exóstoma las esclereidas forman un resalto suplementado con células altas de la exotesta, constituyendo una cresta exostomal anular (Figuras 3O, 7A-C), tan desarrollada que a veces impide ver el exóstoma en vista lateral (Figura 3N).

Arilo. El arilo se presenta en ambos géneros como una excrecencia inserta alrededor del hilo (Figura 3). Es de consistencia carnosa cuando está fresco y membranáceo cuando seco. Es asimétrico, más largo sobre la rafe que sobre los lados de la semilla, puede quedar limitado a la zona rafeal o envolver la semilla casi por completo (Piriqueta taubatensis). El borde del arilo puede ser entero (Figura 3B, E, N), lobulado (incisiones < mitad) (Figuras 3H-I, 9A), o laciniado (incisiones $>$ mitad), (Figura 3L, 9C); ocasionalmente puede tener pequeños pelos simples unicelulares (Figura 9N).

Ontogenia.- En casi todas las especies el arilo es hilar, ya que se origina exactamente en el límite entre funículo y rafe (Figuras 1I, 8A-I, 9B). Se inicia cuando el primordio seminal ha completado su incurvación (Figura 8A-C). Al completarse la formación de los tegumentos, el arilo es un conspicuo rodete de unas 4-5 capas de células que abraza la futura región hilar (Figura 1H-I).

Desarrollo post-fecundación.- El sitio donde aparece el tejido de abscisión de la semilla, es decir el hilo, se ubica sobre una línea imaginaria tangente desde el exóstoma hasta la base del arilo (Figura $8 \mathrm{H}$, ta). El crecimiento laminar del arilo continúa con el desarrollo de la semilla. No hay incremento en espesor, el crecimiento se produce principalmente en los bordes.

En Turnera blanchetiana la inserción del arilo se extiende sobre la rafe, desde el hilo hasta la parte media de la semilla (Figuras 8J, 9O-P). Por su inserción se le define como ráfico. También se presenta en $T$. chrysocephala, T. hermannioides y $T$. joelii.

Anatomía del arilo.- Tiene 5 o 6 estratos de espesor en la base, adelgazándose hacia los bordes, las células son parenquimáticas sin espacios intercelulares, con gran cantidad de granos de almidón compuestos y glóbulos de lípidos. No posee estomas. Las células de la epidermis externa forman una empalizada (Figura 8Q-U); su pared externa puede ser lisa, convexa o papilosa; cada célula puede tener una o varias papilas, dispersas o alineadas (Figura 9E-N). La epidermis interna presenta células alargadas dispuestas longitudinalmente, pequeñas, con cutícula generalmente lisa de menor espesor que la externa.

\section{Discusión}

La anatomía de los óvulos es semejante en todas las especies analizadas, sólo hay variación en el grado de curvatura y en la presencia de células taníferas acompañando el haz ovular en Piriqueta. La ontogenia de los óvulos observada en todas las especies estudiadas concuerda con la descripta por Vijayaraghavan y Kaur (1967) para Turnera ulmifolia. Luego de la fecundación se mantiene el número de estratos celulares; el tegumento externo constituye la testa de la semilla, con dos estratos; el tegumento interno forma el tegmen con tres estratos, por estas características la cubierta seminal se clasifica como no multiplicativa; las semillas se catalogan como exotégmicas porque el exotegmen origina el estrato mecánico (Corner, 1976).

La cubierta seminal tiene una estructura básica integrada por aréolas (determinadas por células gigantes de la endotesta) limitadas por muros (constituidos por las esclereidas del exotegmen). La exotesta puede acompañar este diseño sin sufrir modificaciones, con lo cual la estructura resultante es una cubierta seminal reticulada. Se presentan dos variantes que dependen del desarrollo de los muros transversales. El subtipo reticulado es el más generalizado, se le encuentra en Piriqueta y en varias series de Turnera; el subtipo estriado-reticulado, con muros transversales poco visibles, se presenta en especies de algunas series de Turnera. Cuando las células de la exotesta producen crestas de altura diversa, cubren y enmascaran el diseño básico, constituyendo una cubierta seminal crestada. Este trabajo confirma que también la ontogenia de la cubierta seminal crestada es diferente debido a que la ornamentación es producida principalmente por la exotesta. La cubierta crestada, exclusiva de T. sidoides, es única en la familia, permitiendo identificarla inequívocamente por las semillas. Es la especie de distri- 

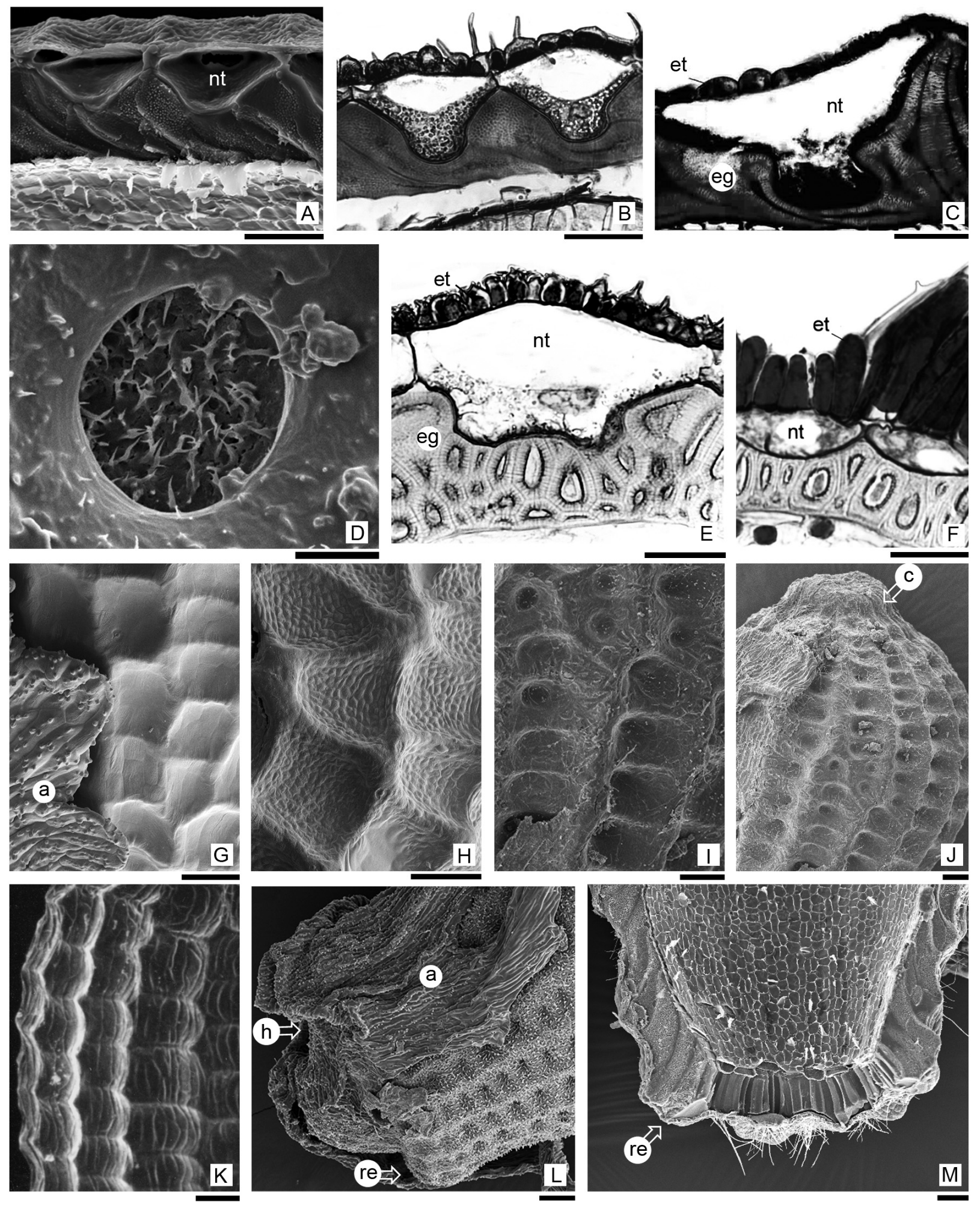

Figura 5. Detalles de la cubierta seminal con microscopía óptica y MEB. A-C. CL de cubierta seminal. A. Turnera capitata. B. Piriqueta morongii. C-D. P racemosa. C. CL cubierta seminal. D. Detalle de fondo de aréola mostrando una depresión punctiforme y paredes laberínticas. E. P. nanuzae, CT de semillas con dos depresiones por aréola. F. T. sidoides subsp. pinnatifida, CT de cubierta seminal crestada. G-L. Superficie de la cubierta seminal (MEB). G. T. chamaedryfolia. H. T. pumilea. I-J. T rubrobracteata. I. Detalle episperma. J. Cálaza. K. P. caiapoensis. L-M. Zona basal en vista superficial y CL mostrando el resalto exostomal. L. P. densiflora. M. T. pumilea. a: arilo; c: cálaza; eg: exotegmen; et: exotesta; h: hilo; nt: endotesta; re: resalto exostomal. Barras: A-F = $50 \mu \mathrm{m} ; \mathrm{D}=20 \mu \mathrm{m}$; G-M = $100 \mu \mathrm{m}$. 

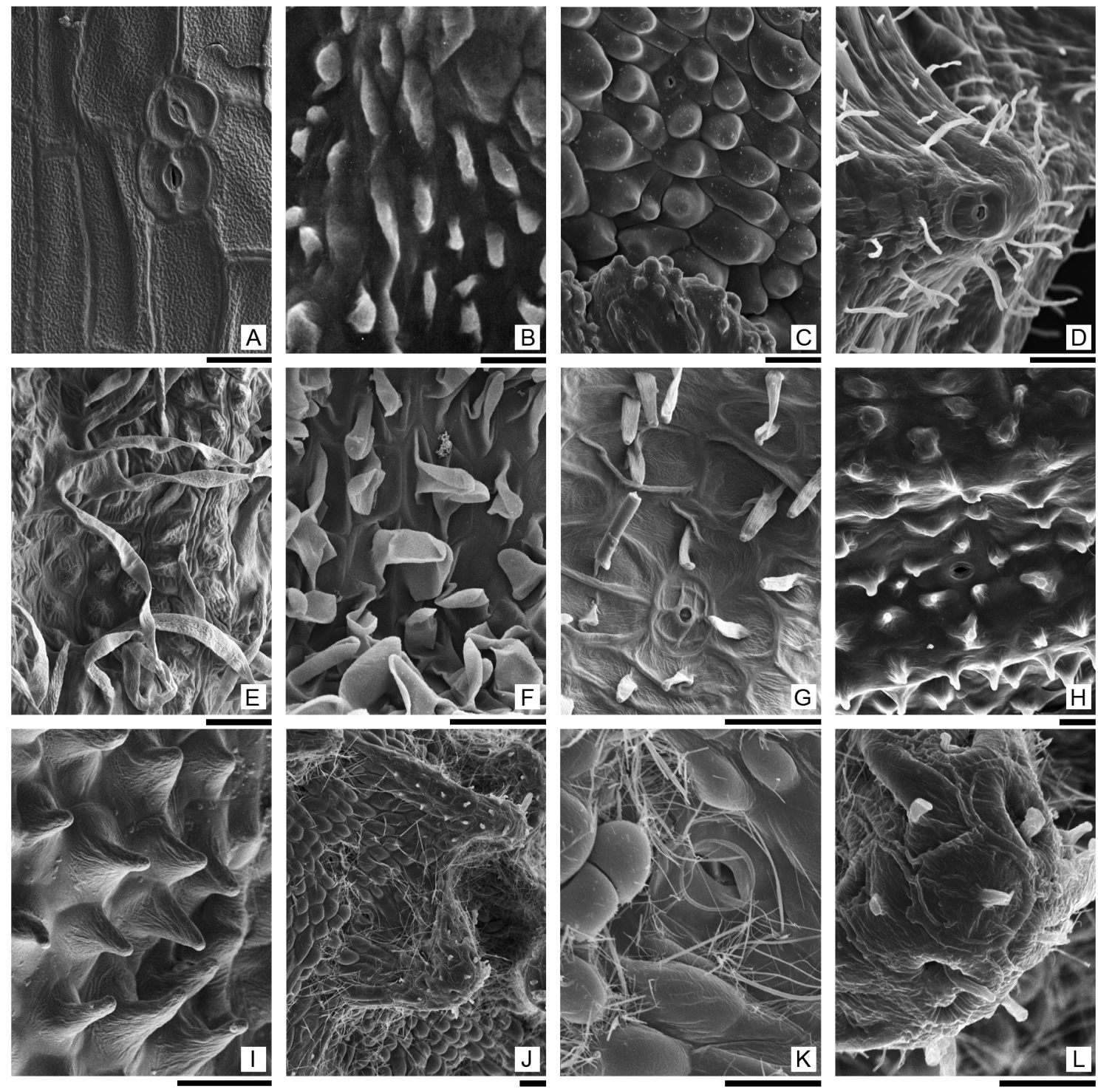

Figura 6. Detalles de la exotesta con MEB. A. Turnera aurelii. B. T. subnuda. C. T. annularis. D. Piriqueta racemosa. E. T. weddelliana. F. T. acuta. G. P. cistoides. H. T. marmorata. I. T. blanchetiana. J-L. T. sidoides subsp. sidoides. Barra $=25 \mu \mathrm{m}$.

bución más austral en el género, y constituye un complejo de cinco subespecies en las que se encuentran niveles de ploidía desde $2 \mathrm{x}$ a $8 \mathrm{x}$. Este taxón está ubicado en la serie Leiocarpae, a pesar de tener caracteres diferentes como el fruto rugoso (Urban, 1883; Arbo, 2008; Gonzalez, 2010).

En Piriqueta, la exotesta de muchas especies presenta papilas digitifomes. En algunas especies se encuentran depresiones punctiformes en las aréolas, solitarias o dobles. La única excepción es $P$. racemosa, con cubierta seminal reticulada de nudos prominentes, depresiones punctiformes muy notorias y papilas filiformes cortas en la exotesta. Esta combinación de caracteres, única en la familia, permite identificar la especie por medio de las semillas.

El género Turnera, el más numeroso de la familia, es el que ofrece mayor diversidad. Sin embargo, los caracteres seminales no son constantes en todas las series del género. Las semillas de la serie Annulares son inconfundibles: cortas, rectas, con aréolas proporcionalmente grandes, exotesta papilosa y cálaza abultada. En las series Microphyllae y Papilliferae son glabras, con retículo notorio y cálaza obtusa, igual que en Turnera subserie Turnera. En cambio, en Turnera subserie Umbilicatae presentan retículo poco perceptible y cálaza prominente, umbilicada. En las series Anomalae y Salicifoliae y en varias especies de Stenodictyae se asocian la cubierta seminal estriado-reticulada, la exotesta con papilas filiformes y la cálaza umbilicada. Varias especies de la serie Capitatae presentan una cubierta seminal reticulada, exotesta papilosa y cálaza umbilicada.

Hay caracteres que sólo se encuentran en especies de la serie Leiocarpae, como la cubierta seminal reticulada con 


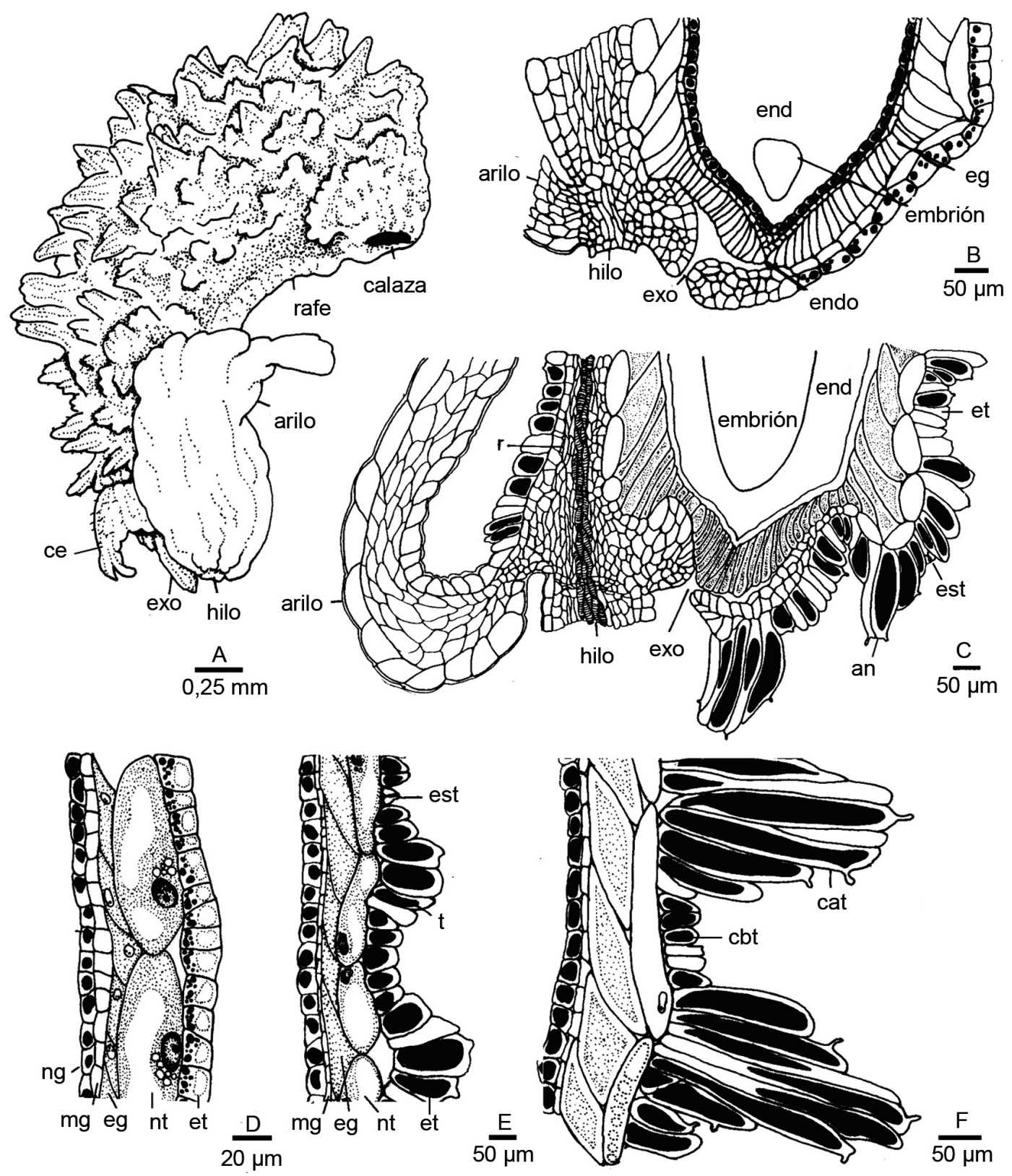

Figura 7. Semilla de Turnera sidoides subsp. pinnatifida. A. semilla madura. B-C. Detalle de la región de la micrópila joven y madura respectivamente. D-F. Sucesivos estados de desarrollo de la cubierta seminal. cat: células altas taníferas; cbt: células bajas taníferas; ce: cresta exostomal anular; eg: exotegmen; end: endosperma; endo: endóstoma; est: estoma; et: exotesta; exo: exóstoma; mg: mesotegmen; ng: endotegmen; nt: endotesta; t: taninos.

nudos prominentes (Turnera cipoensis, T. guianensis, T. nervosa, T. pumilea (Figura 3D), T. trigona (Figura 3F), ) o con bastones de cera epicuticular (T. argentea, T. lamiifolia, T. longiflora, T. melochioides, T. nervosa, T. opifera, T. pumilea (Figura 5M), T. sidoides (Figura 6K), T. subnuda). En algunas especies la exotesta presenta células grandes sobre los muros y células pequeñas papilosas sobre las aréolas $(T$. argentea, T. callosa, T. dasytricha, T. dolichostigma, T. genistoides, T. hassleriana, T. melochioides, T. subnuda).
La proliferación dérmica en el ápice de la rafe es notoria en especies de la serie Leiocarpae (Turnera acaulis, T. callosa, T. dasytricha (Figura 3E), T. discolor, T. dolichostigma, T. hassleriana, T. lamiifolia, T. trigona (Figura 3F), T. longiflora), pero excepcionalmente aparece en otras series (T. dichotoma, Figura 3M) y en Piriqueta.

Corner (1976), siguiendo a Baillon (1876), usa el término "arilo" para los apéndices carnosos de las semillas, originados en el óvulo o el funículo. Este trabajo aplica esa de- 

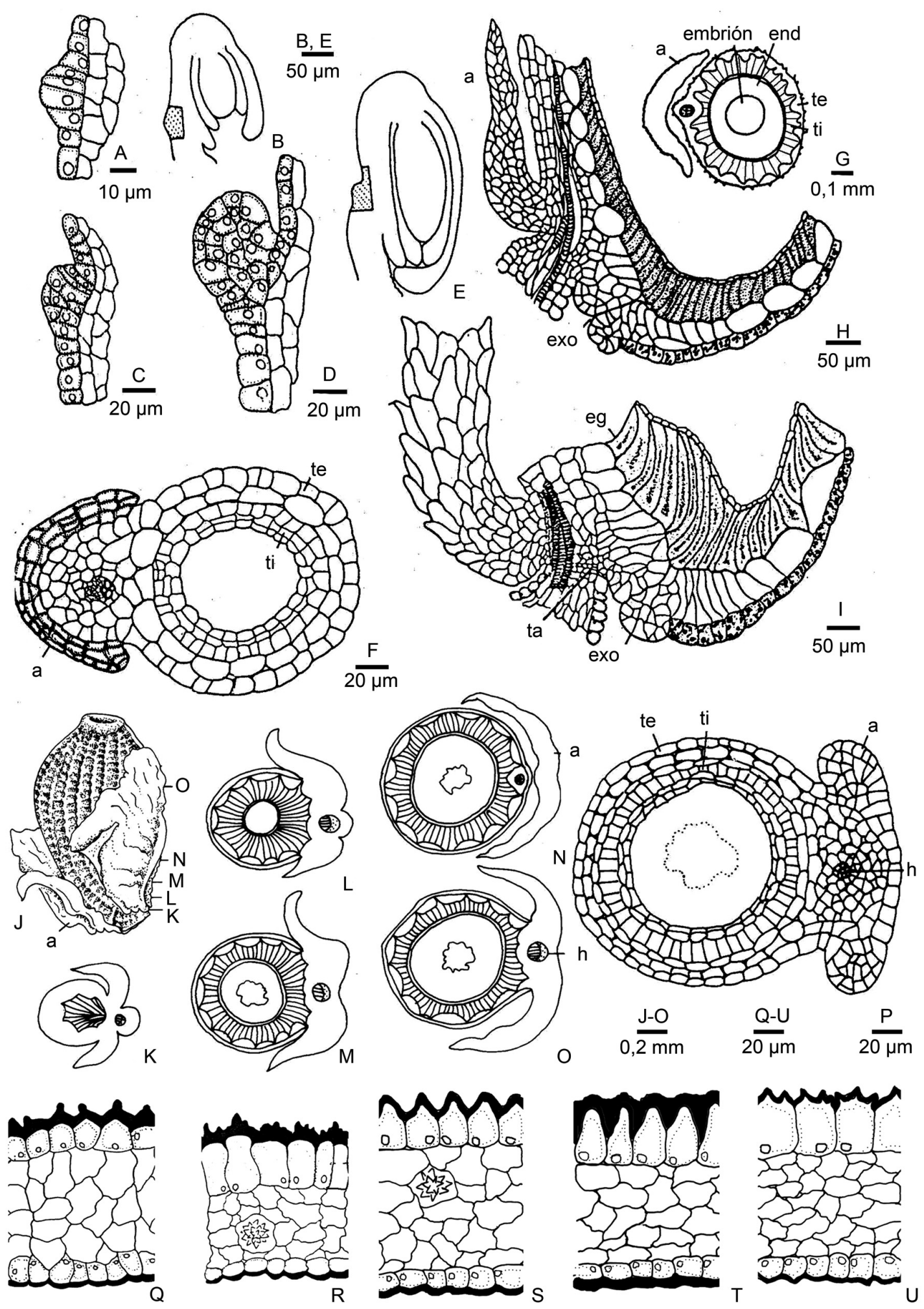

Figura 8. Arilo. A-I. Turnera aurelii. A. inicio del arilo en CL de óvulo, correspondiente a B. C-D. CL mostrando sucesivos estadios del desarrollo del arilo, correspondientes a E. F. CT de óvulo al momento de la antesis, realizado a la altura del arilo. G. CT de semilla. H-I. CL de semilla joven y madura, mostrando el desarrollo del arilo. J-O. T. blanchetiana. J. Semilla madura. K-O. CT por los distintos niveles indicados en J, mostrando la inserción del arilo. P. CT de óvulo. Q-U. CT de arilos en semillas maduras. Q. Piriqueta racemosa. R. P. morongii. S. T. cuneiformis. T. T. melochioides. U. T. chamaedrifolia. a: arilo; c: cálaza; eg: exotegmen; end: endosperma; ex: exóstoma; h: hacecillo de la rafe; ta: tejido de absición; te: tegumento externo; ti: tegumento interno. 

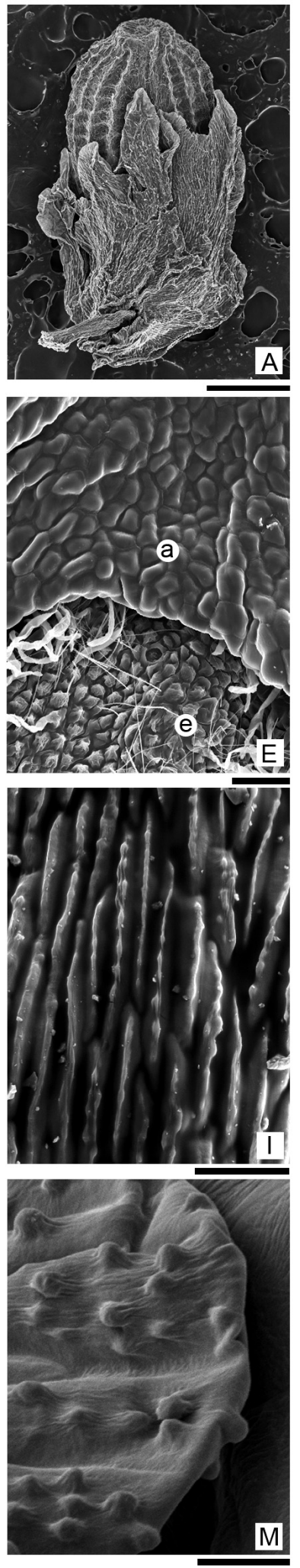
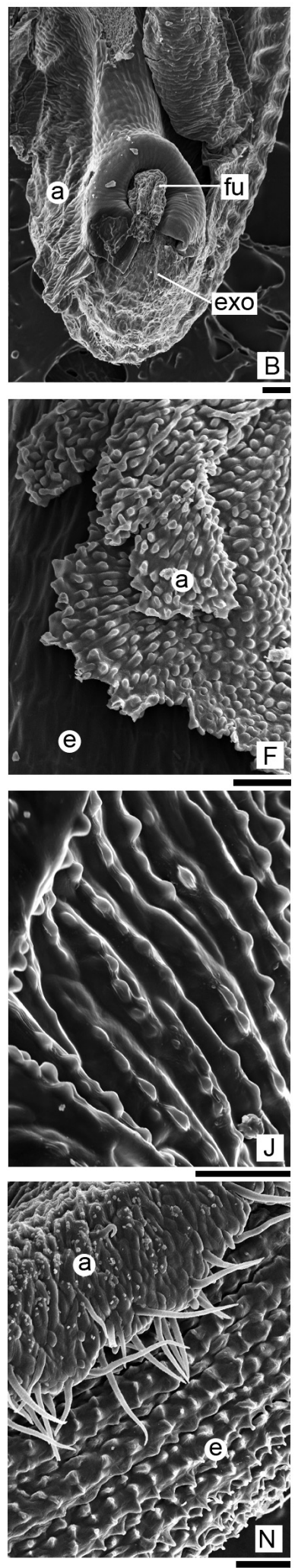
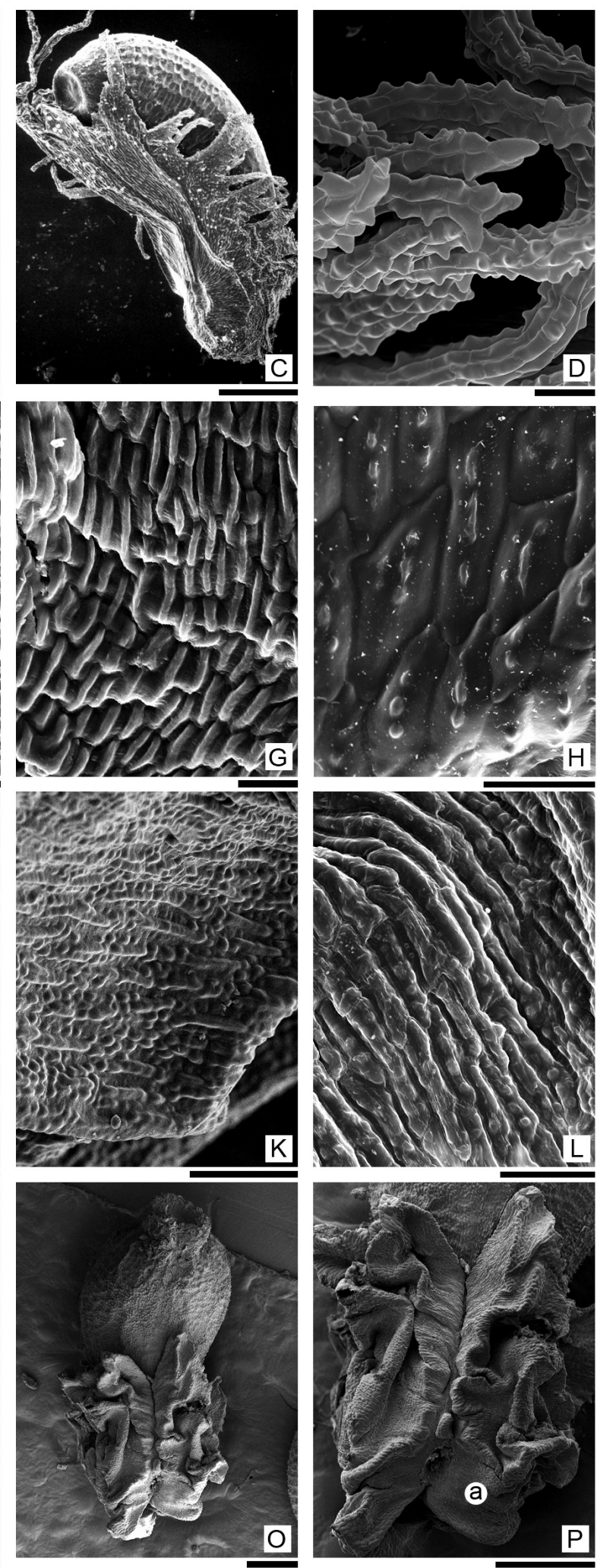
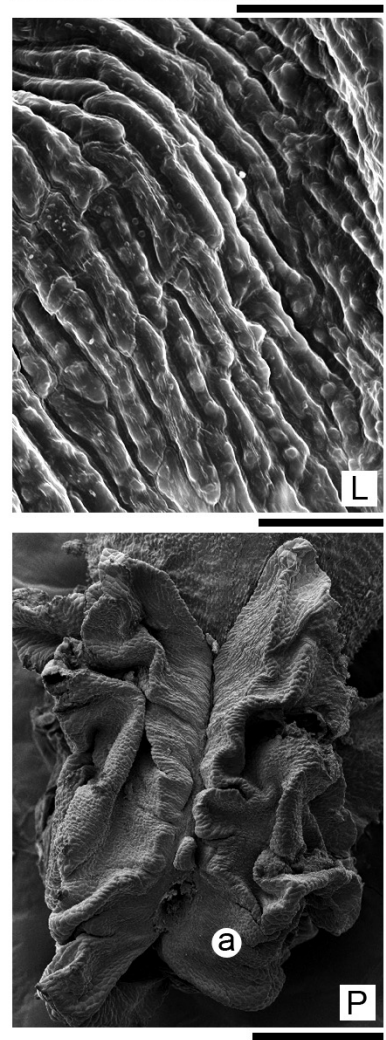

Figura 9. Arilo (MEB). A. Turnera capitata. B. T. luetzerburgii, detalle de la zona micropilar. C-D. T. stenophylla, C. Semilla, D. Detalle de borde de arilo. E. T. weddelliana. F-N. Superficie del arilo. F. T. arcuata. G. T. incana. H. T. stenophylla. I. Piriqueta ocroleuca. J. T. diffusa. K. T. trigona. L. T. sidoides. M. T. chamaedryfolia. N. T. marmorata. O-P. T. blanchetiana, O. Semilla, P. Arilo. a: arilo; e: cubierta seminal; exo: exóstoma; fu: funículo. Barras: A, C, O-P = 500 $\mu \mathrm{m} ; \mathrm{B}, \mathrm{N}=100 \mu \mathrm{m} ; \mathrm{D}, \mathrm{L}=50 \mu \mathrm{m} ; \mathrm{M}=20 \mu \mathrm{m}$. 
finición, y califica el arilo según su origen en hilar o ráfico, según Baillon (1876). El hilo es la cicatriz que deja en la semilla la abscisión del funículo, y se define la rafe como la adnación del funículo al rudimento seminal (Werker, 1997). En el presente estudio se confirma, anatómica y ontogenéticamente, que en las especies estudiadas de Piriqueta y en la gran mayoría de las especies de Turnera la inserción del arilo es hilar (Arbo, 1995, 2008). Dos especies de la serie Anomalae (T. blanchetiana, T. chrysocephala) y dos de la subserie Umbilicatae (T. hermannioides y $T$. joelii) presentan arilo ráfico. Si el arilo fuera funicular (Corner, 1976) se desprendería al producirse su abscisión de la semilla. En todas las especies estudiadas se confirma el origen dermal del arilo. En ninguna especie analizada se observaron las capas hipodérmicas que menciona Raju (1956) para T. ulmifolia. El crecimiento laminar del arilo se produce después de la fecundación.

Con el análisis micromorfológico y anatómico del arilo de numerosas especies, este trabajo aporta datos que permiten diferenciar especies similares; como por ejemplo Turnera concinna y T. krapovickasii. Los resultados de este estudio demuestran que los caracteres seminales, solos o combinados, permiten la individualización de ciertas especies, grupos de especies o algunas series de Turnera, y ayudan a caracterizar Piriqueta. En el Cuadro 1 se presentan los caracteres anatómicos seminales que se destacan por su valor diagnóstico.

\section{Agradecimientos}

A los revisores por las valiosas sugerencias realizadas al manuscrito. A las instituciones patrocinantes de los viajes de colección de material botánico: CONICET y UNNE.

\section{Literatura citada}

Arbo M.M. 1995. Turneraceae. Parte I. Piriqueta. Flora Neotrópica Monograph 67:1-157.

Arbo M.M. 1997. Estudios sistemáticos en Turnera (Turneraceae) I. Series Salicifoliae y Stenodictyae. Bonplandia 9:151-208.

Arbo M.M. 2000. Estudios sistemáticos en Turnera (Turneraceae) II. Series Annulares, Capitatae, Microphyllae y Papilliferae. Bonplandia 10:1-82.

Arbo M.M. 2005. Estudios sistemáticos en Turnera (Turneraceae). III. Series Anomalae y Turnera. Bonplandia 14:115-318

Arbo M.M. 2008. Estudios sistemáticos en Turnera (Turneraceae). IV. Series Leiocarpae, Conciliatae y Sessilifoliae. Bonplandia 17:107-334.

Bahadur B., Rama Swamy N. y Srikanth R. 1981. The taxonomic significance of seed and aril characters (LM \& SEM) in two species of Turnera L. (Turneraceae). Indian Journal of Botany 4:209-215.
Baillon H.E. 1876. Sur l'origine du macis de la muscade et des arilles en général. Adansonia 11:329-340.

Barthlott W. 1981. Epidermal and seed surface characters of plants: systematic applicability and some evolutionary aspects. Nordic Journal of Botany 1:345-355.

Barthlott W. 1984. Microstructural features of seed surface. En: Heywood V.H. y Moore D.M. Eds. Current Concepts in Plant Taxonomy, pp. 95-105, Academic Press, Londres.

Berger M.G. 1919. Etude organographique, anatomique et pharmacologique des Turnéracées. Thése $n^{\circ} 58$. Faculté de Médecine et de Pharmacie de Lille, París.

Corner E.J.H. 1976. The Seeds of Dicotyledons. Vol. 2. Cambridge University Press, Cambridge.

D’Ambrogio de Argüeso A. 1986. Manual de Técnicas en Histología Vegetal. Ed. Hemisferio Sur, Buenos Aires.

Gonzalez A.M. 1993. Anatomía y vascularización floral de Piriqueta racemosa, Turnera hassleriana y Turnera joelii (Turneraceae). Bonplandia 7:143-184.

Gonzalez A.M. 2010. Anatomía y desarrollo del fruto en Piriqueta y Turnera (Turneraceae). Boletín de la Sociedad Argentina de Botánica 45:257-272.

Gonzalez A.M. y Cristóbal C.L. 1997. Anatomía y ontogenia de semillas de Helicteres lhotzkyana (Sterculiaceae). Bonplandia 9:287-294.

Grande J.R., Kallunki J., Arbo M.M., Berry P.E., Huber O. y Riina R. 2012. Botanical novelties from Sierra de Maigualida, Southern Venezuela. II. Anales del Jardín Botánico de Madrid 69:7-19.

Johansen D.A. 1940. Plant Microtechnique. McGraw-Hill Book Company, Nueva York.

Kloos A. y Bouman F. 1980. Case studies in aril development. Passiflora suberosa L. and Turnera ulmifolia L. Beitrage zur Biologie der Pflanzen 55:49-66.

Luque R., Sousa H.C. y Kraus J.E. 1996. Métodos de coloração de Roeser (1972) - modificado - e Kropp (1972) visando a substituição do azul de astra por azul de alcião 8 GS ou 8 GX. Acta Botânica Brasílica 10:199-212.

Munsell. 1977. Munsell Color Charts for Plant Tissues. 2nd ed. Munsell Color Company, Baltimore.

Raju M.V.S. 1956. Development of embryo and seed coat in Turnera ulmifolia L. var. angustifolia Willd. Botaniska Notiser 109:308-312

Ruzin S.E. 1999. Plant Microtechnique and Microscopy. Oxford University Press, Nueva York.

Thulin M., Razafimandimbison S.G., Chafe P., Heidari N., Kool A. y Shore J.S. 2012. Phylogeny of the Turneraceae clade (Passifloraceae s.1.): Trans-Atlantic disjunctions and two new genera in Africa. Taxon 61:308-323.

Urban I. 1883. Monographie der familie der Turneraceen. Gebrüder Borntraeger, Berlín.

Vijayaraghavan M.R. y Kaur D. 1967. Morphology and embryology of Turnera ulmifolia L. and affinities of the family Turneraceae. Phytomorphology 16:539-553.

Werker E. 1997. Seed Anatomy. Volume 10, Part 3 of Handbuch der Pflanzenanatomie Encyclopedia of Plant Anatomy - Traite d' Anatomie Vegetale Series. Gebrüder Borntraeger, Berlín.

Recibido: 28 de diciembre de 2012

Aceptado: 13 de abril de 2013 
Semillas en TuRnera y PirIQUeta.

Apéndice. Lista de especies estudiadas. Los ejemplares de las especies utilizadas se encuentran en el herbario del Instituto de Botánica del Nordeste (CTES), Corrientes, Argentina, salvo se indique otra cosa. Abreviaturas: F: material fijado en FAA, C: mantenido bajo cultivo, $\mathrm{H}$ : ejemplar de herbario.

\section{Piriqueta}

Piriqueta caiapoensis Arbo. Brasil: Goiás: ca. 50 Km of Caiaponia on road to Jataí, Irwin \& Soderstrom 7378 (P), H. Piriqueta carnea Urb. Brasil: Bahia: alrededores de Santo Inácio, Arbo et al. 5330, F. Piriqueta cistoides (L.) Griseb. subsp. caroliniana (Walt.) Arbo. Brasil: Bahia: laçu, Hatschbach et al. 56981, C. Piriqueta densiflora Urb. var. densiflora. Brasil: Bahia: alrededores de Santo Inácio, Arbo et al. 5322, F. Bahia: 20 km S de Xique-Xique, Arbo et al. 7490, H. Piriqueta duarteana (Cambess.) Urb. var. duarteana. Brasil: Bahia: BR-242, 18 km W de la entrada a Lençóis, Arbo et al. 5312, H. Bahia: 19 Km NW de Jacobina, BR324, Arbo et al. 7386, H. Piriqueta morongii Rolfe. Argentina: Corrientes: Ituzaingó, Villa Olivari, Gonzalez 32, C. Corrientes: ruta 12 y entrada a Sta. Ana. Arbo et al. 6040, C. Piriqueta nanuzae Arbo. Brasil: Bahia: Mucujé. Arbo et al. 2540, F. Piriqueta ochroleuca Urb. Paraguay: Cordillera: Caacupé, barrio Kennedy. Gonzalez et al. 17, F. Piriqueta racemosa (Jacq.) Sw. Brasil: Bahia: 1 km N de Baixa Grande. Krapovickas et al. 38807 bis, C. Piriqueta sidifolia (Cambess.) Urb. var. multiflora Urb. Brasil: Minas Gerais: Buenópolis, Curimataí, Mello Silva et al. 614, F. Minas Gerais: Agroindustrial Serra do Cabral, Hatschbach et al. 64873, H. Piriqueta suborbicularis (A.St.-Hil. \& Naudin) Arbo. Paraguay: Itapúa: 9 km SE de General Delgado. Arbo et al. 6155, C y Gonzalez et al. 28, C. Itapúa: 9 km NW de Cnel. Bogado, Gonzalez et al. 29, C. Piriqueta taubatensis (Urb.) Arbo. Argentina: Misiones: Puerto Candelaria, Loreto, Schinini et al. 27589, C. Misiones: Ayo. Zaimán, Gonzalez 33, C. Corrientes: Ituzaingó, ruta 34, camino a San Carlos, Gonzalez 39, C.

\section{Turnera}

Serie Annulares. Turnera annularis Urb. Brasil: Bahia: ca. $10 \mathrm{~km}$ na entrada a ca. $1 \mathrm{~km}$ na estrada para Itacaré, Jardim 1032, F. Turnera aromatica Arbo. Belize: 30-38 mi along Hummingbird highway S of Belmopan, Croat 24816, H. Turnera breviflora Moura. Venezuela: faldas de Ávila, Williams \& Alston 48 (NY), H. Turnera odorata Rich. Brasil: Amapá: Rio Oiapoque, Irwin et al. $47413, \mathrm{H}$.

Serie Anomalae. Turnera blanchetiana Urb. Brasil: Bahia: $15 \mathrm{~km}$ al W de Itiúba, Arbo et al. 7323, F. Bahia: BA-426, $7 \mathrm{~km} \mathrm{~S} \mathrm{de}$ Varzéa Nova, Arbo et al. 7434, H. Turnera cearensis Urb. Brasil: Bahia, $6 \mathrm{~km}$ from Filadelfia on the BA-385 to Itiuba, Harley et al. 16164, H. Turnera laciniata Arbo. Brasil: Pará: estrada Santarem-Cuiaba, BR-163, km 886 a 881, Amaral 1152, H. Brasil: Pará: $6 \mathrm{~km}$ SE of AMZA, Sperling et al. 5764, H.

Serie Capitatae. Turnera albicans Urb. Brasil: Bahia: estrada antiga, 2-4 Km W de Sta. Cruz Cabralia, Mori et al. 10924, H. Turnera capitata Cambess. Brasil: São Paulo: Parque Estadual das Fontes de Ipiranga, Catharino \& Gonzalez 2154, F. Minas Gerais: Serra da Mantiqueira, Hatsbach 45579, H.

Serie Conciliatae. Turnera rubrobracteata Arbo. Brasil: Espírito Santo: Margens do Rio Doce, Pedra do Feijoal, Kuhlmann 6656, H.

Serie Leiocarpae. Turnera argentea Arbo. Venezuela: Amazonas: al E del Caño Perro de Agua afluente derecho del Rio Orinoco, 30 Km SW de confluencia Orinoco-Ventuari, Huber \& Tillet 2809, H. Turnera cipoensis Arbo. Brasil: Minas Gerais: km 127 da rodovia Lagõa Santa-Conceição do Mato Dentro, Semir \& Sazima 494, H. Minas Gerais: Serra do Cipó, Pereira 8897, H. Turnera coccinea Arbo. Brasil: Minas Gerais: BR-251, extremo N de serra da Bocaina, próx. a las nacientes del rio Ventania, Mello Silva et al. 760, F. Turnera dasytricha Pilger. Bolivia: Santa Cruz: San Ignacio, $10 \mathrm{Km}$ hacia N, Bruderreck 346, H. Turnera discolor Urb. Brasil: Mato Grosso: Braço, Rio Arinos, Baldwin 3035, H. Turnera genistoides Cambess. Brasil: Minas Gerais: Diamantina, estrada para Biribiri, Simão-Bianchini 89, H. Turnera guianensis Aubl. Venezuela: Bolívar: Cerro Baraguán and vicinity, Wurdack \& Monachino 40205, H. Turnera hassleriana Urb. Paraguay: Concepción: 12 km NE de Loreto, Vanni et al. 395, C. Paraguarí: 2 km E de ruta Piribebuy-Paraguarí, sobre el desvío a Itá-Morotí y Valenzuela, Arbo et al. 6082, C. Paraguarí: Chololó, Gonzalez et al. 22, F. Turnera lamiifolia Cambess. Brasil: Minas Gerais: N of Grão Mogol, Irwin et al. 23418, H. Brasilia: Parque Nacional, Krapovickas, Cristóbal \& Arbo 3168, H. Turnera Iongiflora Cambess. Brasil: Goiás, 13 km by road S of São João da Aliança, Anderson 7580, H. Turnera melochioides Cambess. Brasil: Bahia: BR-324, 12 km NW a Jacobina, Arbo et al. 7372, H. Paraguay: Amambay: Parque Nac. Cerro Corá, Gonzalez et al. 55, F. Turnera nervosa Urb. Argentina: Corrientes: Ituzaingó, Rincón Ombú Chico, Arbo 2076, C. Turnera opifera Mart. Brasil: Minas Gerais: 16 km de Itacambira estrada a Pau-d'Óleo, Mello Silva et al. 636, F. Minas Gerais: Botumirim, Mello Silva et al. 641, F. Turnera pumilea L. Brasil: Piauí: 3 km W de Oeiras, Krapovickas et al. 38624 bis, C. Turnera sidoides L. subsp. carnea (Cambess.) Arbo. Uruguay: Cerro Largo: Bañado Medina, ruta 44, km 409, Solís Neffa et al. 271, C. Turnera sidoides L. subsp. integrifolia (Griseb.) Arbo. Argentina: Entre Ríos: Colón, puerto, Solís Neffa et al. 310, C. Turnera sidoides L. subsp. pinnatifida (Juss. ex Poir.) Arbo. Argentina: Corrientes: ruta 123, $5 \mathrm{~km}$ E de la entrada a Yofre, 
Apéndice. Continuación

Solís Neffa et al. 307, C. Jujuy: Ledesma, ruta 34, 4 km S de Fraile Pintado, Schinini 19532, F. Jujuy: El Carmen, Pampa Blanca, Schinini 19536, F. Turnera sidoides L. subsp. sidoides. Uruguay: Maldonado, R.12, camino de San Carlos a Minas, Solis Neffa et al. 547, C. Turnera subnuda Urb. Brasil: Goiás: Alrededores de Colinas, sobre camino a Cavalcante, 600-700 m, Arbo et al. 3677, F. Turnera trigona Urb. Brasil: Goiás: 39 km NW de Nova Roma, Arbo et al. 3564, F.

Serie Microphyllae. Turnera collotricha Arbo. Brasil: Minas Gerais: Mun. Gouveia, Serra do Espinhaço, Hatschbach et al. 36434, H. Turnera diffusa Willd ex Schultes. Brasil: Bahia: 14 km WNW de Morro do Chapéu, Arbo et al. 5354, F. Bahia: 19 km NW de Jacobina, BR-324, Arbo et al. 7394, H. Mexico: Puebla, Pringle 6692, H. Turnera hebepetala Urb. Brasil: Bahia: Barauninha, Hatschbach et al. 65059, F.

Serie Papillifera. Turnera caatingana Arbo. Brasil: Bahia: $20 \mathrm{~km} \mathrm{E} \mathrm{de} \mathrm{Caetité,} \mathrm{camino} \mathrm{a} \mathrm{Brumado,} \mathrm{Arbo} \mathrm{et} \mathrm{al.} \mathrm{5645,} \mathrm{F.} \mathrm{Bahia:}$ BR-030, 7 km E de Caetité, camino a Brumado, Arbo et al. 7643, H. Turnera chamaedrifolia Cambess. Brasil: Bahia: Feira de Santana, Noblick 3175 bis, C.

Serie Salicifoliae. Turnera brasiliensis Willd. ex Schultes. Brasil: Pará: Região do Rio Capim, Rio Candirú-Açú, Fróes 33620, H. Turnera glaziovii Urb. Guayana Francesa: de St.Laurent a Paul Isnard, entre la route d'Apatou et la Crique Portal, Granville 5166, H. Turnera hindsiana Benth. Ecuador: Los Ríos: entre Mocachi y Palenque on the Estero Peñafiel, Gentry 30717, H. Turnera panamensis Urb. Panamá: Panamá, Shore s/n, F. Turnera rupestris Aubl. Guayana Francesa: Sinnamary: Saut Vata, Grenaud 1709, H. Turnera serrata Vell. Brasil: Paraná: Cerradinho, Hatschbach et al. 51907, H. Turnera venosa Urb. Brasil: Amazonas, río Abacaxis, Terra Preta, Hills et al. 12934, H. Turnera weddelliana Urb. \& Rolfe. Paraguay: Paraguarí: Cerro Capilla, Gonzalez et al. 25, C.

Serie Sessilifoliae. Turnera dichotoma Gardner. Brasil: Minas Gerais: São Gonçalo do Rio Preto junto ao côrrego da Lapa, Lombardi et al. 3724, H. Minas Gerais: Guinda, 1200-1300 m, Hatschbach 27369, H. Turnera revoluta Urb. Brasil: Minas Gerais: $11,4 \mathrm{~km}$ sudoeste da estrada Congonhas do Norte - Gouveia, entrada a 3,7 km NW de Congonhas do Norte. Mello-Silva et al. $2402, \mathrm{H}$.

Serie Stenodictyae. Turnera acuta Willd. ex Schultes. Colombia: Guainia: outskirts of San Felipe, Gentry y Stein 46477, H. Perú: Loreto: Mainas, Iquitos, Pto. Almendras, Vázquez \& Jaramillo 10236, H. Venezuela: Amazonas: Mun. Autana, Río Cuao, entre Raudal del Danto y Caño la Raya, Castillo 5476, F. Turnera aurantiaca Benth. Brasil: Corantyne river near Wonotobo, Schultz $7711, \mathrm{H}$.

Serie Turnera Subserie Turnera. Turnera aurelii Arbo. Paraguay: Río Salado: camino de Limpio a Emboscada, Schinini 23860, C. Turnera grandiflora (Urb.) Arbo. Argentina: Corrientes: Dep. Saladas, cruce rutas 12 y 17, Solís Neffa et al. 42, H. Turnera scabra Millsp. Argentina: Corrientes, Arbo y Fernández 2757, H.

Serie Turnera Subserie Umbilicatae. Turnera coriacea Urb. Brasil: Minas Gerais: Serra do Cipó, 10-20 km NE de Cardeal Mota, Arbo et al. 4187, H. Turnera hermannioides Cambess. Brasil: Bahia: about $2 \mathrm{~km} \mathrm{~N}$ of Vila do Rio de Contas, Harley 15618, H. Turnera joelii Arbo. Brasil: Bahia: río Juremal, Barrett \& Shore 1373, C. Turnera leptosperma Urb. Bahia: alrededores de Santo Inácio y hasta $9 \mathrm{Km}$ al N, Arbo et al. 5344, H. Turnera stenophylla Urb. Brasil: Bahia: alrededores de Santo Inácio y hasta $9 \mathrm{~km}$ al N, Arbo et al. 5319, F. Bahia: 20 km S de Xique-Xique, Arbo et al. 7488, H. 